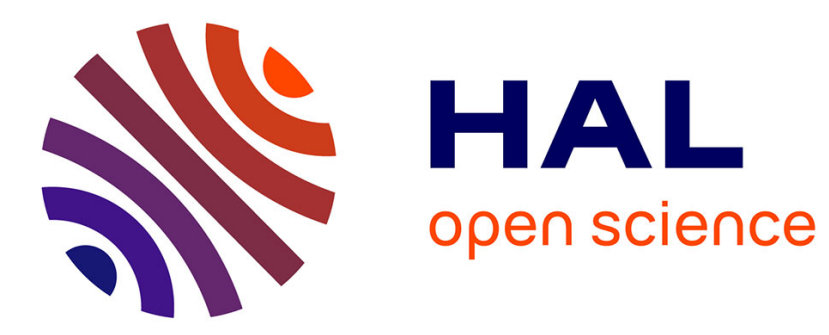

\title{
On the energy-minimizing strains in martensitic microstructures-Part 1: Geometrically nonlinear theory
}

Michaël Peigney

\section{To cite this version:}

Michaël Peigney. On the energy-minimizing strains in martensitic microstructures-Part 1: Geometrically nonlinear theory. Journal of the Mechanics and Physics of Solids, 2013, 61, pp.1489-1510. 10.1016/j.jmps.2012.12.009 . hal-00813543

\section{HAL Id: hal-00813543 \\ https://hal-enpc.archives-ouvertes.fr/hal-00813543}

Submitted on 18 Sep 2015

HAL is a multi-disciplinary open access archive for the deposit and dissemination of scientific research documents, whether they are published or not. The documents may come from teaching and research institutions in France or abroad, or from public or private research centers.
L'archive ouverte pluridisciplinaire HAL, est destinée au dépôt et à la diffusion de documents scientifiques de niveau recherche, publiés ou non, émanant des établissements d'enseignement et de recherche français ou étrangers, des laboratoires publics ou privés. 


\title{
On the energy-minimizing strains in martensitic microstructures - Part 1: Geometrically nonlinear theory
}

\author{
Michaël Peigney \\ Université Paris-Est, Laboratoire Navier (Ecole des Ponts ParisTech, IFSTTAR, \\ CNRS), F-77455 Marne-la-Vallée Cedex 2, France
}

\begin{abstract}
This paper addresses the theoretical prediction of the set of energyminimizing strains that can be realized by martensitic microstructures. Polyconvexification and related notions are used to derive some upper bounds (in the sense of inclusion of sets). Lower bounds are constructed by lamination techniques. The geometrically nonlinear theory (finite strains) is considered in the present Part 1. Analytical expressions are obtained for a three-well problem which encompasses the cubic to tetragonal transformation as a special case. Twelve-well problems related to cubic to monoclinic transformations are also studied. In that case, sufficient conditions are derived for the microstructure to be restricted to only two of the 12 wells.
\end{abstract}

Keywords: phase transformation nonlinear bounds relaxation finite strain

\section{Introduction}

Some metallic alloys exhibit a solid/solid phase transformation between different crystallographic structures, known as austenite (stable at high temperature) and martensite (stable at low temperature). That phase transformation can be triggered both by thermal and mechanical loading. In terms of crystallographic structure, the austenite has a higher symmetry than the martensite. This leads one to distinguish several symmetry-related martensitic variants. Those variants correspond to different orientations of the martensitic lattice with respect to the austenitic lattice. Accordingly, to each martensitic variant is attached a transformation strain, describing the deformation between the crystallographic structures of the austenite and 
the martensite. The number of martensitic variants as well as the corresponding transformation strains depends on the alloy considered, through the structure of the austenite and martensite lattices. Some common examples include the cubic to tetragonal transformation $(\mathrm{MnCu}, \mathrm{MnNi})$, the cubic to orthorombic transformation $\left(\beta_{1}^{\prime} \mathrm{CuAlNi}\right)$ and the cubic to monoclinic transformations (NiTi, $\gamma_{1}^{\prime} \mathrm{CuAlNi}$ ), corresponding respectively to 3,6 and 12 martensitic variants.

The phase transformation between austenite and martensite gives rise to the shape memory effect displayed by alloys such as NiTi or CuAlNi: cooling down a stress-free sample below a critical temperature transforms the homogeneous austenite to a martensitic microstructure, in which the martensitic variants arrange themselves so as to produce a stress-free state with no macroscopic deformation. This phenomenon is classically referred to as self-accommodation. Deforming the sample entails a reorientation of the variants, i.e. a phase transformation of some martensitic variants to others. After unloading the sample, a residual stress-free strain is observed at the macroscopic level. That residual strain results from the cooperative effect of the microscopic transformation strains in each variant. Heating the sample transforms the martensite back in austenite, thus restoring the initial configuration.

The shape memory effect is obviously limited: if for instance the strain imposed in the cooled state is too large, plastic deformations will occur and the material will no longer be able to recover its initial shape after heating. This motivates the definition of recoverable strains as macroscopic strains that can be restored by the shape memory effect. As explained by Bhattacharya and Kohn (1997), recoverable strains can be interpreted as minimizers of the macroscopic energy at low temperature. That macroscopic energy is itself obtained as the relaxation of a multi-well energy function $\Psi$ that models the behavior of the material at a microscopic level. The relaxation procedure is notoriously difficult to perform and essentially consists in finding the austenite/martensite microstructures which minimize the global energy. This paper is essentially concerned with the theoretical prediction of the set of strains that minimize the macroscopic (or effective) energy, using the framework of nonlinear elasticity.

The problem can be formulated either in the geometrically nonlinear setting or in the geometrically linear setting. The geometrically nonlinear setting is more accurate and therefore to be preferred, especially as uniaxial measurements show that recoverable strains may be of the order of $10 \%$. It 
turns out, however, that the problem is significantly more tractable in the geometrically linear setting, which in turn allows the analysis to be pushed further. For three-dimensional problems, exact solutions are available only in few cases. In the geometrically non-linear theory, Ball and James (1992) solved the case of two compatible variants with the same determinant. Bhattacharya and Dolzmann (2001) extended that solution to a special case of the four-well problem, which remains two-dimensional in nature. In the geometrically linear theory, the solution of the two-well problem has been obtained by Kohn (1991). Smyshlyaev and Willis (1998) developed the approach of Kohn (1991) and adapted it to the three-well problem, deriving a lower bound on the relaxed energy and giving a sufficient condition for that lower bound to be realizable.

This paper aims at complementing existing results on that problem, essentially through the use of bounds on the set of energy-minimizing strains. Part 1 is devoted to the geometrically nonlinear theory, whereas the geometrically linear theory is considered in Part 2 (Peigney, 2013). In Part 1 we are particularly interested in studying the three-well problem, in the geometrically nonlinear theory. The outline of the present Part 1 is as follows. In Section 2 is derived a general upper bound based on distinctive properties of Young measures (Kinderlehrer and Pedregal, 1991; Ball and James, 1992; Müller, 1999). Lower bounds are obtained using sequential lamination techniques (Kohn, 1991; Ball and James, 1992; Dolzmann, 1999; Stupkiewicz and Petryk, 2002). To that purpose, the solution of the two-well problem plays an essential role and therefore is recalled in Section 3. Most of the results in Section 3 are already known, but for the sake of comprehensiveness they are reported explicitly and stated as theorems for latter reference. The two-well problem also serves as a first illustrative example of the methodology introduced in Section 2. Building on the results of Section 2 and 3, a three-well problem is studied in detail in Section 4. Much emphasis is put on the cubic to tetragonal transformation, which is a special case of the three-well problem considered. Closed-form expressions of upper and lower bounds on the set of energy-minimizing strains are obtained and compared. Section 5 focuses on cubic to monoclinic transformations: using results of Section 2, we give sufficient conditions on the macroscopic deformation for the microstructure to involve only two of the 12 variants, extending similar studies carried out by Ball and James (1992) and Bhattacharya et al. (1999) for the cubic to tetragonal and cubic to orthorombic transformations, respectively. 


\section{Upper bounds on $Q \mathcal{K}$}

At the microscopic level, the free energy density $\Psi$ of martensitic crystals is classically modeled as a multi-well function of the form $\min _{1 \leq r \leq n+1} \Psi_{r}$ where $\Psi_{r}$ is the free energy of phase $r$ and $n$ is the number of martensitic variants. We label the phases so that $r=n+1$ corresponds to the austenite, and $1 \leq r \leq n$ corresponds to the martensite variants. Each free energy $\Psi_{r}$ is a function of the deformation gradient $\boldsymbol{F}$ and is frame indifferent, i.e. satisfies $\Psi_{r}(\boldsymbol{R F})=\Psi_{r}(\boldsymbol{F})$ for all $\boldsymbol{R} \in \mathrm{SO}(3)$ and for all $\boldsymbol{F}$. Moreover, the free energies $\Psi_{r}$ of the martensite variants are symmetry related, i.e. for each $1 \leq r \leq n$ there exists a rotation $\boldsymbol{R}_{r}$ such that

$$
\Psi_{r}(\boldsymbol{F})=\Psi_{1}\left(\boldsymbol{R}_{r} \boldsymbol{F} \boldsymbol{R}_{r}^{T}\right) \text { for all } \boldsymbol{F} .
$$

Let us denote by $\mathcal{K}$ the set of deformation gradients $\boldsymbol{F}$ that minimize the function $\Psi$. The property (2.1) immediately shows that $\min \Psi_{r}=\min \Psi_{1}$ for all $r \leq n$, so that $\min \Psi=\min \left\{\min \Psi_{1}, \min \Psi_{n+1}\right\}$. At a temperature below the transformation temperature, martensite achieves the minimum energy, i.e. $\min \Psi_{n+1}>\min \Psi_{1}$. In such case, the set $\mathcal{K}$ is given by

$$
\mathcal{K}=\bigcup_{r=1}^{n} \mathcal{K}_{r}
$$

where $\mathcal{K}_{r}=\left\{\boldsymbol{F} \mid \Psi_{r}(\boldsymbol{F})=\min \Psi_{r}\right\}$. The frame indifference of $\Psi_{r}$ implies that $\mathcal{K}_{r}$ can be written as $\mathcal{K}_{r}=\mathrm{SO}(3) \mathcal{U}_{r}$ where $\mathcal{U}_{r}$ is a set of symmetric definite positive tensors. More specifically, for martensitic crystals, the set $\mathcal{K}_{r}$ assumes the form

$$
\mathcal{K}_{r}=\mathrm{SO}(3) \boldsymbol{U}_{r}
$$

where the distinct symmetric positive definite tensors $\left\{\boldsymbol{U}_{r}\right\}_{1 \leq r \leq n}$ are the transformation strains of the different variants. Note from (2.1) that the strains $\left\{\boldsymbol{U}_{r}\right\}$ are symmetry related, i.e. $\boldsymbol{U}_{r}$ can be written as $\boldsymbol{U}_{r}=\boldsymbol{R}_{r} \boldsymbol{U}_{1} \boldsymbol{R}_{r}^{T}$, where $\boldsymbol{R}_{r}$ is the rotation appearing in (2.1).

Consider now a martensitic single crystal occupying a domain $\Omega$ in the reference configuration. We denote by $\boldsymbol{x} \mapsto \boldsymbol{u}(\boldsymbol{x})$ the mapping between the reference configuration and a deformed configuration at equilibrium. The crystal is subjected to boundary conditions of the form $\boldsymbol{u}(\boldsymbol{x})=\overline{\boldsymbol{F}} \cdot \boldsymbol{x}$ where $\overline{\boldsymbol{F}}$ is a given effective deformation gradient. In the equilibrium theory of 
martensitic transformations, the starting assumption is that the system minimizes its effective energy, as defined by

$$
Q \Psi(\overline{\boldsymbol{F}})=\inf _{\boldsymbol{F} \in \mathcal{A}(\overline{\boldsymbol{F}})} \frac{1}{|\Omega|} \int_{\Omega} \Psi(\boldsymbol{F}) d \boldsymbol{x}
$$

where

$$
\begin{array}{r}
\mathcal{A}(\overline{\boldsymbol{F}})=\left\{\boldsymbol{F} \mid \operatorname{det} \boldsymbol{F}>0, \exists \boldsymbol{u}(\boldsymbol{x}) \in W^{1, \infty}\left(\Omega, \mathbb{R}^{3}\right)\right. \text { such that } \\
\boldsymbol{F}=\nabla \boldsymbol{u} \text { in } \Omega ; \boldsymbol{u}(\boldsymbol{x})=\overline{\boldsymbol{F}} . \boldsymbol{x} \text { on } \partial \Omega\} .
\end{array}
$$

Since the function $\Psi$ is bounded from below, the quantity $Q \Psi(\overline{\boldsymbol{F}})$ is finite. However, since $\Psi$ is generally not quasiconvex, minimizing sequences do not necessarily converge: there may not be existence of a gradient field $\boldsymbol{F}^{0}$ in $\mathcal{A}(\overline{\boldsymbol{F}})$ such that $Q \Psi(\overline{\boldsymbol{F}})=(1 /|\Omega|) \int_{\Omega} \Psi\left(\boldsymbol{F}^{0}\right) d \boldsymbol{x}$. This property corresponds physically to the formation of microstructures at a fine scale. Such microstructures can be described mathematically using Young measures (see e.g. Kinderlehrer and Pedregal (1991) and references therein). The function $\overline{\boldsymbol{F}} \mapsto Q \Psi(\overline{\boldsymbol{F}})$ is classically referred to as the quasiconvexification (or relaxation) of the function $\Psi$.

In this paper we focus on the set $Q \mathcal{K}$ of deformation gradients that minimize the effective free energy, i.e.

$$
Q \mathcal{K}=\{\overline{\boldsymbol{F}} \mid Q \Psi(\overline{\boldsymbol{F}})=\min Q \Psi\}
$$

As proved by Ball and James (1992), $Q \mathcal{K}$ is the set of deformation gradients $\overline{\boldsymbol{F}}$ for which there exists a bounded sequence $\boldsymbol{F}_{j}$ in $\mathcal{A}(\overline{\boldsymbol{F}})$ verifying $\operatorname{dist}\left(\boldsymbol{F}_{j}, \mathcal{K}\right) \rightarrow 0$ in measure in $\Omega$. That last characterization shows that $Q \mathcal{K}$ only depends on $\Psi$ through the set $\mathcal{K}$.

The set $Q \mathcal{K}$ in (2.6) is related to the set of gradient Young measures by the following theorem :

Theorem 1. (Ball and James, 1992; Müller, 1999) For any $\overline{\boldsymbol{F}} \in Q \mathcal{K}$, there exists a gradient Young measure $\nu$ supported on $\mathcal{K}$ such that $\overline{\boldsymbol{F}}=$ $\int_{\mathbb{R}^{3 \times 3}} \boldsymbol{A} d \nu(\boldsymbol{A})$.

In the following, that theorem is used to derive upper bounds on $Q \mathcal{K}$. Recall that the gradient Young measures (supported on $\mathcal{K}$ ) that appear in Theorem 1 have the following properties (Kinderlehrer and Pedregal, 1991):

$$
\begin{gathered}
\nu \geq 0, \int_{\mathbb{R}^{3 \times 3}} d \nu(\boldsymbol{A})=1, \\
h\left(\int_{\mathbb{R}^{3 \times 3}} \boldsymbol{A} d \nu(\boldsymbol{A})\right) \leq \int_{\mathbb{R}^{3 \times 3}} h(\boldsymbol{A}) d \nu(\boldsymbol{A}) \text { for any } h \text { quasiconvex. }
\end{gathered}
$$


A function $h$ is quasiconvex if

$$
|\Omega| h(\overline{\boldsymbol{F}}) \leq \int_{\Omega} h(\boldsymbol{F}) d \boldsymbol{x} \text { for all } \overline{\boldsymbol{F}} \text { and } \boldsymbol{F} \in \mathcal{A}(\overline{\boldsymbol{F}}) .
$$

We refer to the textbooks of Müller (1999) and Dacorogna (2008) for a comprehensive study of Young measures and quasiconvexity. Let us now examine how upper bounds on $Q \mathcal{K}$ can be obtained from the properties mentioned above. For any quasiconvex function $h$ and any $\overline{\boldsymbol{F}}$ in $Q \mathcal{K}$, the conjunction of Theorem 1 with (2.7) implies that

$$
h(\overline{\boldsymbol{F}}) \leq \sup _{\mathcal{K}} h .
$$

Let $\mathcal{H}$ be a given family of quasiconvex functions and define

$$
P_{0} \mathcal{K}=\left\{\overline{\boldsymbol{F}} \mid 0 \geq \sup _{h \in \mathcal{H}}\left[h(\overline{\boldsymbol{F}})-\sup _{\mathcal{K}} h\right]\right\} .
$$

It follows from (2.9) that $Q \mathcal{K} \subset P_{0} \mathcal{K}$, i.e. $P_{0} \mathcal{K}$ is an upper bound (in the sense of inclusion of sets) on $Q \mathcal{K}$.

Observe that the particular structure $(2.2)$ of $\mathcal{K}$ has not been taken into account so far: the decomposition (2.2-2.3) shows that $\mathcal{K}=\cup_{r=1}^{n} \mathcal{K}_{r}$ where the wells $\mathcal{K}_{r}=\mathrm{SO}(3) \boldsymbol{U}_{r}$ are closed and disjoint. In that case (see lemma 3.3 in Ball and James (1992)), the Young measure $\nu$ can be written as

$$
\nu=\sum_{r=1}^{n} \nu_{r}
$$

where $\nu_{r}$ is a positive measure supported on $\mathcal{K}_{r}$. For any $\overline{\boldsymbol{F}}$ in $Q \mathcal{K}$ and $h$ in $\mathcal{H}$, we now obtain that

$$
h(\overline{\boldsymbol{F}}) \leq \sum_{r=1}^{n} \int_{\mathbb{R}^{3 \times 3}} h(\boldsymbol{A}) d \nu_{r}(\boldsymbol{A}) \leq \sum_{r=1}^{n} \theta_{r} \sup _{\mathcal{K}_{r}} h
$$

where

$$
\theta_{r}=\int_{\mathbb{R}^{3 \times 3}} d \nu_{r}(\boldsymbol{A})
$$

can be interpreted as the volume fraction of phase $r$ in the microstructures corresponding to the Young measure $\nu$. Note that $\boldsymbol{\theta}=\left(\theta_{1}, \cdots, \theta_{n}\right)$ must belong to the set $\mathcal{T}_{n}$ defined as

$$
\mathcal{T}_{n}=\left\{\boldsymbol{\theta}=\left(\theta_{1}, \cdots, \theta_{n}\right) \mid \theta_{r} \geq 0 ; \sum_{r=1}^{n} \theta_{r}=1\right\}
$$


It follows from (2.11) that $Q \mathcal{K}$ is included in the set $P \mathcal{K}$ defined by

$$
P \mathcal{K}=\bigcup_{\boldsymbol{\theta} \in \mathcal{T}_{n}} P \mathcal{K}(\boldsymbol{\theta})
$$

where

$$
P \mathcal{K}(\boldsymbol{\theta})=\left\{\overline{\boldsymbol{F}} \mid 0 \geq \sup _{h \in \mathcal{H}}\left[h(\overline{\boldsymbol{F}})-\sum_{r=1}^{n} \theta_{r} \sup _{\mathcal{K}_{r}} h\right]\right\} .
$$

A given family $\mathcal{H}$ of quasiconvex functions thus generates two upper bounds on $\mathcal{K}$, namely the bound $P_{0} \mathcal{K}$ in (2.10) and the bound $P \mathcal{K}$ given in (2.13). Only the latter makes use of the special structure $(2.2)$ of $\mathcal{K}$. It can be easily checked that $P \mathcal{K} \subset P_{0} \mathcal{K}$, but the reverse inclusion is not necessarily true (an example is given in Section 4.1).

The relations (2.12-2.13) also provide some information on the microstructures that realize a given deformation gradient $\overline{\boldsymbol{F}}$ in $Q \mathcal{K}$. In such microstructures, the volume fractions $\boldsymbol{\theta}$ of the different wells must necessarily be such that $\overline{\boldsymbol{F}} \in P \mathcal{K}(\boldsymbol{\theta})$. Therefore, for a given $\overline{\boldsymbol{F}}$, the set

$$
\{\boldsymbol{\theta} \mid \overline{\boldsymbol{F}} \in P \mathcal{K}(\boldsymbol{\theta})\}
$$

is an upper bound on the possible volume fractions. This gives some partial information on the underlying microstructure, although not as refined as the full knowledge of the Young measure.

Let us now turn our attention to the choice of a family $\mathcal{H}$ of quasiconvex functions to be used in the calculation of $P_{0} \mathcal{K}$ and $P \mathcal{K}$. A direct consequence of the decomposition (2.2) is that $Q \mathcal{K}$ respects the frame indifference, i.e.

$$
\overline{\boldsymbol{F}} \in Q \mathcal{K} \Longrightarrow \mathrm{SO}(3) \overline{\boldsymbol{F}} \subset Q \mathcal{K} \text {. }
$$

Therefore it is a natural requirement that the upper bound considered also respects the frame indifference. Using the definitions (2.10) and (2.13), it can easily be verified that $P_{0} \mathcal{K}$ and $P \mathcal{K}$ are frame indifferent if $\mathcal{H}$ satisfies the following property:

$$
\text { for all } \boldsymbol{R} \in S O(3) \text { and } h \in \mathcal{H} \text {, the function } \boldsymbol{F} \mapsto h(\boldsymbol{R} . \boldsymbol{F}) \text { is in } \mathcal{H} \text {. }
$$

In principle the best bounds available from (2.10) and (2.13) are obtained by optimizing with respect to all quasiconvex functions, which in practice is not 
possible because there is no simple characterization of quasiconvexity. In the following, we restrict our attention to functions of the form

$$
h(\boldsymbol{F})=\boldsymbol{\tau}: \boldsymbol{F}+\boldsymbol{a}: \boldsymbol{F}^{*}+c \operatorname{det} \boldsymbol{F}
$$

where $\boldsymbol{\tau}, \boldsymbol{a}$ and $c$ are arbitrary, and $\boldsymbol{F}^{*}=\operatorname{det} \boldsymbol{F}\left({ }^{T} \boldsymbol{F}\right)^{-1}$ is the adjugate of $\boldsymbol{F}$. The functions (2.16) are null-lagrangians, i.e. (2.8) holds as an equality. Moreover, the property (2.15) is respected by that family of functions. With this particular choice of quasiconvex functions, the bound $P_{0} \mathcal{K}$ in $(2.10)$ consists of tensors $\overline{\boldsymbol{F}}$ such that

$$
0 \geq \sup _{\boldsymbol{\tau}, \boldsymbol{a}, c}\left\{\overline{\boldsymbol{F}}: \boldsymbol{\tau}+\boldsymbol{a}: \overline{\boldsymbol{F}}^{*}+c \operatorname{det} \overline{\boldsymbol{F}}-\sup _{\boldsymbol{F} \in \mathcal{K}}\left(\boldsymbol{F}: \boldsymbol{\tau}+\boldsymbol{a}: \boldsymbol{F}^{*}+c \operatorname{det} \boldsymbol{F}\right)\right\}
$$

while the bound $P \mathcal{K}(\boldsymbol{\theta})$ in $(2.13)$ consists of tensors $\overline{\boldsymbol{F}}$ such that

$0 \geq \sup _{\boldsymbol{\tau}, \boldsymbol{a}, c}\left\{\overline{\boldsymbol{F}}: \boldsymbol{\tau}+\boldsymbol{a}: \overline{\boldsymbol{F}}^{*}+c \operatorname{det} \overline{\boldsymbol{F}}-\sum_{r=1}^{n} \theta_{r} \sup _{\boldsymbol{F} \in \mathcal{K}_{r}}\left(\boldsymbol{F}: \boldsymbol{\tau}+\boldsymbol{a}: \boldsymbol{F}^{*}+c \operatorname{det} \boldsymbol{F}\right)\right\}$.

The bound $P_{0} \mathcal{K}$ given by $(2.17)$ is classically refered to as the polyconvexification (or polyconvex hull) of $\mathcal{K}$ (see Dacorogna (2008) and references therein). Using (2.2) and taking $\boldsymbol{\tau}=\boldsymbol{a}=0$ in (2.18), we can see that any $\overline{\boldsymbol{F}}$ in $P \mathcal{K}(\boldsymbol{\theta})$ satisfies

$$
\operatorname{det} \overline{\boldsymbol{F}}=\sum_{r} \theta_{r} \operatorname{det} \boldsymbol{U}_{r}
$$

For any $\overline{\boldsymbol{F}}$ satisfying this condition, the requirement (2.18) is equivalent to

$$
0 \geq \sup _{\boldsymbol{\tau}, \boldsymbol{a}}\left\{\overline{\boldsymbol{F}}: \boldsymbol{\tau}+\boldsymbol{a}: \overline{\boldsymbol{F}}^{*}-\sum_{r=1}^{n} \theta_{r} \sup _{\boldsymbol{F} \in \mathcal{K}_{r}}\left(\boldsymbol{F}: \boldsymbol{\tau}+\boldsymbol{a}: \boldsymbol{F}^{*}\right)\right\}
$$

For solving the maximization problem over $\mathcal{K}_{r}$ that appears in $(2.20)$, it is convenient to introduce the function $\Phi$ defined for any second-order tensor $M$ by

$$
\Phi(\boldsymbol{M})=\sup _{\boldsymbol{S} \in \mathrm{SO}(3)} \boldsymbol{S}: \boldsymbol{M}
$$

The function $\Phi(\boldsymbol{M})$ can be calculated using the polar decomposition of $\boldsymbol{M}$. We can indeed write $\boldsymbol{M}=\boldsymbol{R} . \boldsymbol{V}$ where $\boldsymbol{R} \in \mathrm{SO}(3)$ and $\boldsymbol{V}$ is either symmetric 
positive (if $\operatorname{det} \boldsymbol{M} \geq 0$ ) or symmetric negative (if $\operatorname{det} \boldsymbol{M}<0$ ). Denoting by $\lambda_{1}, \lambda_{2}, \lambda_{3}$ the eigenvalues of $\boldsymbol{V}$, it can be shown (Peigney, 2008) that

$$
\Phi(\boldsymbol{M})=f\left(\lambda_{1}, \lambda_{2}, \lambda_{3}\right)
$$

where $f$ is the positive function defined on $\mathbb{R}^{3}$ by

$$
f(x, y, z)= \begin{cases}|x|+|y|+|z| & \text { if } x y z \geq 0 \\ |x|+|y|+|z|-2 \min (|x|,|y|,|z|) & \text { if } x y z<0 .\end{cases}
$$

Using the relation $\mathcal{K}_{r}=\mathrm{SO}(3) \boldsymbol{U}_{r}$, some elementary manipulations show that $\sup _{\boldsymbol{F} \in \mathcal{K}_{r}}\left(\boldsymbol{F}: \boldsymbol{\tau}+\boldsymbol{a}: \boldsymbol{F}^{*}\right)=\Phi\left(\boldsymbol{U}_{r} . \boldsymbol{\tau}+\boldsymbol{U}_{r}^{*} \cdot \boldsymbol{a}\right)$. The bound $P \mathcal{K}(\boldsymbol{\theta})$ therefore consists of deformation gradients $\overline{\boldsymbol{F}}$ verifying (2.19) and

$$
0 \geq \sup _{\boldsymbol{\tau}, \boldsymbol{a}}\left\{\overline{\boldsymbol{F}}: \boldsymbol{\tau}+\boldsymbol{a}: \overline{\boldsymbol{F}}^{*}-\sum_{r=1}^{n} \theta_{r} \Phi\left(\boldsymbol{U}_{r} \cdot \boldsymbol{\tau}+\boldsymbol{U}_{r}^{*} \cdot \boldsymbol{a}\right)\right\} .
$$

The bound $P_{0} \mathcal{K}$ in $(2.17)$ is found to consist of deformation gradients $\overline{\boldsymbol{F}}$ verifying $\operatorname{det} \overline{\boldsymbol{F}}=\max _{r} \operatorname{det} \boldsymbol{U}_{r}$ and

$$
0 \geq \sup _{\boldsymbol{\tau}, \boldsymbol{a}}\left\{\overline{\boldsymbol{F}}: \boldsymbol{\tau}+\boldsymbol{a}: \overline{\boldsymbol{F}}^{*}-\max _{1 \leq r \leq n} \Phi\left(\boldsymbol{U}_{r} \cdot \boldsymbol{\tau}+\boldsymbol{U}_{r}^{*} \cdot \boldsymbol{a}\right)\right\}
$$

For a given $\overline{\boldsymbol{F}}$, it is in general necessary to resort to numerical techniques for solving the optimization problem in (2.24) or (2.25) over all tensors $(\boldsymbol{\tau}, \boldsymbol{a})$ in $\mathbb{R}^{3 \times 3} \times \mathbb{R}^{3 \times 3}$. Analytical bounds on $Q \mathcal{K}$ can be obtained by restricting $(\boldsymbol{\tau}, \boldsymbol{a})$ to a well chosen class $\mathcal{C}$ of tensors for which the optimization can be performed in closed-form. Such a class of tensors depends on the problem considered, notably on the transformation strains $\boldsymbol{U}_{r}$. Using (2.15), it can be verified that the corresponding bound will respect the frame indifference provided that

$$
\forall \boldsymbol{R} \in \mathrm{SO}(3),(\boldsymbol{\tau}, \boldsymbol{a}) \in \mathcal{C} \Longrightarrow(\boldsymbol{a} . \boldsymbol{R}, \boldsymbol{\tau} . \boldsymbol{R}) \in \mathcal{C} .
$$

A possible choice is to consider tensors $(\boldsymbol{\tau}, \boldsymbol{a})$ of the form

$$
\left(\boldsymbol{v} \otimes \boldsymbol{w}, \boldsymbol{v}^{\prime} \otimes \boldsymbol{w}\right)
$$

where $\boldsymbol{v}, \boldsymbol{v}^{\prime}$ and $\boldsymbol{w}$ are arbitrary vectors. Let us now explicit the corresponding restrictions on $Q \mathcal{K}$. Using (2.24) we obtain

$$
0 \geq \boldsymbol{w} \cdot \overline{\boldsymbol{F}} \cdot \boldsymbol{v}+\boldsymbol{w} \cdot \overline{\boldsymbol{F}}^{*} \cdot \boldsymbol{v}^{\prime}-\sum_{r=1}^{n} \theta_{r} \Phi\left(\left(\boldsymbol{U}_{r} \cdot \boldsymbol{v}+\boldsymbol{U}_{r}^{*} \cdot \boldsymbol{v}^{\prime}\right) \otimes \boldsymbol{w}\right)
$$


The relation (2.22) gives $\Phi\left(\left(\boldsymbol{U}_{r} . \boldsymbol{v}+\boldsymbol{U}_{r}^{*} . \boldsymbol{v}^{\prime}\right) \otimes \boldsymbol{w}\right)=\|\boldsymbol{w}\|\left\|\boldsymbol{U}_{r} . \boldsymbol{v}+\boldsymbol{U}_{r}^{*} . \boldsymbol{v}^{\prime}\right\|$, so that (2.28) becomes

$$
0 \geq \boldsymbol{w} \cdot \overline{\boldsymbol{F}} \cdot \boldsymbol{v}+\boldsymbol{w} \cdot \overline{\boldsymbol{F}}^{*} \cdot \boldsymbol{v}^{\prime}-\|\boldsymbol{w}\|\left(\sum_{r=1}^{n} \theta_{r}\left\|\boldsymbol{U}_{r} \cdot \boldsymbol{v}+\boldsymbol{U}_{r}^{*} \cdot \boldsymbol{v}^{\prime}\right\|\right)
$$

That inequality holds for any vectors $\boldsymbol{v}, \boldsymbol{v}^{\prime}$ and $\boldsymbol{w}$. For fixed values of $\boldsymbol{\theta}, \overline{\boldsymbol{F}}$, $\boldsymbol{v}$ and $\boldsymbol{v}^{\prime}$, the right-hand side of (2.29) is maximized when $\boldsymbol{w}$ is parallel to $\overline{\boldsymbol{F}} . \boldsymbol{v}+\overline{\boldsymbol{F}}^{*} . \boldsymbol{v}^{\prime}$, giving

$$
\left\|\overline{\boldsymbol{F}} . \boldsymbol{v}+\overline{\boldsymbol{F}}^{*} \cdot \boldsymbol{v}^{\prime}\right\| \leq \sum_{r=1}^{n} \theta_{r}\left\|\boldsymbol{U}_{r} . \boldsymbol{v}+\boldsymbol{U}_{r}^{*} . \boldsymbol{v}^{\prime}\right\| .
$$

For any $\overline{\boldsymbol{F}}$ in $Q \mathcal{K}$, there exists $\boldsymbol{\theta}$ in $\mathcal{T}_{n}$ such that (2.19) and (2.30) are satisfied. Note that use of the tensors (2.27) in (2.25) gives the following upper bound on $Q \mathcal{K}$ :

$$
\left\|\overline{\boldsymbol{F}} \cdot \boldsymbol{v}+\overline{\boldsymbol{F}}^{*} \cdot \boldsymbol{v}^{\prime}\right\| \leq \max _{1 \leq r \leq n}\left\|\boldsymbol{U}_{r} \cdot \boldsymbol{v}+\boldsymbol{U}_{r}^{*} \cdot \boldsymbol{v}^{\prime}\right\|
$$

where $\left(\boldsymbol{v}, \boldsymbol{v}^{\prime}\right)$ are arbitrary vectors. The relations (2.30) and (2.31) will be frequently used in the following. In general, those relations do not contain all the information included in (2.24), as tensors $(\boldsymbol{\tau}, \boldsymbol{a})$ of the form (2.27) do not attain the supremum in (2.24) or (2.25). Therefore the relations (2.30)(2.31) will be complemented by other restrictions obtained by optimizing (2.24) with respect to other tensors $(\boldsymbol{\tau}, \boldsymbol{a})$ than those of the form $(2.27)$.

\section{A two-well reference problem}

In the geometrically nonlinear setting, the exact expression of $Q \mathcal{K}$ has been obtained by Ball and James (1992) for a special case of the two-well problem. The corresponding solution applies to the situation where $\mathcal{K}=$ $\mathrm{SO}(3) \boldsymbol{U}_{1} \cup \mathrm{SO}(3) \boldsymbol{U}_{2}$ and $\left(\boldsymbol{U}_{1}, \boldsymbol{U}_{2}\right)$ are symmetric positive tensors such that

(i) $\operatorname{det} \boldsymbol{U}_{1}=\operatorname{det} \boldsymbol{U}_{2}$,

(ii) $\mathrm{SO}(3) \boldsymbol{U}_{1}$ and $\mathrm{SO}(3) \boldsymbol{U}_{2}$ are rank-1 connected.

We recall that two wells $\mathrm{SO}(3) \boldsymbol{U}_{1}$ and $\mathrm{SO}(3) \boldsymbol{U}_{2}$ are said to be rank-1 connected if there exists a rotation $\boldsymbol{R}$ and a unit vector $\boldsymbol{n}$ such that

$$
\boldsymbol{R} \boldsymbol{U}_{2}-\boldsymbol{U}_{1}=\boldsymbol{a} \otimes \boldsymbol{n}
$$

for some vector $\boldsymbol{a}$. For latter reference, we note the following useful characterization of the rank-1 connection between two wells: 
Theorem 2. (Ball and James, 1987) Let $\lambda_{1} \leq \lambda_{2} \leq \lambda_{3}$ be the eigenvalues of $\boldsymbol{U}_{1}^{2}-\boldsymbol{U}_{2}^{2}$. Then $S O(3) \boldsymbol{U}_{1}$ and $S O(3) \boldsymbol{U}_{2}$ are rank-1 connected if and only if $\lambda_{2}=0$.

Let us consider the case $\mathcal{K}^{0}=\mathrm{SO}(3) \boldsymbol{U}_{1}^{0} \cup \mathrm{SO}(3) \boldsymbol{U}_{2}^{0}$ corresponding to

$$
\boldsymbol{U}_{1}^{0}=\left(\begin{array}{ccc}
\eta_{2} & 0 & 0 \\
0 & \eta_{1} & 0 \\
0 & 0 & \eta_{3}
\end{array}\right), \boldsymbol{U}_{2}^{0}=\left(\begin{array}{ccc}
\eta_{1} & 0 & 0 \\
0 & \eta_{2} & 0 \\
0 & 0 & \eta_{3}
\end{array}\right)
$$

where $\eta_{1}, \eta_{2}$ and $\eta_{3}$ are positive parameters. The matrix representations are relative to a reference orthonormal basis $\left(\boldsymbol{v}_{1}, \boldsymbol{v}_{2}, \boldsymbol{v}_{3}\right)$. Any two-well problem satisfying (3.1) can actually be cast under the form (3.3) using a change of variables introduced by Ball and James (1992) and recalled in Appendix A

Since $Q \mathcal{K}^{0}$ respects the principle of frame indifference, it can be decomposed as

$$
Q \mathcal{K}^{0}=\mathrm{SO}(3) \mathcal{U}^{0}
$$

where $\mathcal{U}^{0}$ is included in the set $M_{s}^{3 \times 3}$ of symmetric positive tensors. Consider a fixed $\overline{\boldsymbol{U}}$ in $\mathcal{U}^{0}$. The condition $\operatorname{det} \overline{\boldsymbol{U}}=\eta_{1} \eta_{2} \eta_{3}$ implies that

$$
\overline{\boldsymbol{U}}^{*}=\eta_{1} \eta_{2} \eta_{3} \overline{\boldsymbol{U}}^{-1}
$$

Applying (2.31) with $\left(\boldsymbol{v}, \boldsymbol{v}^{\prime}\right)=\left(\eta_{1} \eta_{2} \boldsymbol{v}_{3},-\eta_{3} \boldsymbol{v}_{3}\right)$, we obtain that $\eta_{3} \overline{\boldsymbol{U}}^{*}{ }^{\boldsymbol{v}_{3}}=$ $\eta_{1} \eta_{2} \overline{\boldsymbol{U}} \cdot \boldsymbol{v}_{3}$, which using (3.5) can be rewritten as $\overline{\boldsymbol{U}}^{2} \boldsymbol{v}_{3}=\eta_{3}^{2} \boldsymbol{v}_{3}$. Since $\overline{\boldsymbol{U}}$ is symmetric positive, it follows directly that $\overline{\boldsymbol{U}} \cdot \boldsymbol{v}_{3}=\eta_{3} \boldsymbol{v}_{3}$, and that $\overline{\boldsymbol{U}}$ admits a matrix representation of the form

$$
\left(\begin{array}{ccc}
u_{11} & u_{12} & 0 \\
u_{12} & u_{22} & 0 \\
0 & 0 & \eta_{3}
\end{array}\right)
$$

The condition $\operatorname{det} \overline{\boldsymbol{U}}=\eta_{1} \eta_{2} \eta_{3}$ and the use of (2.31) with $\left(\boldsymbol{v}, \boldsymbol{v}^{\prime}\right)=\left(\boldsymbol{v}_{1} \pm \boldsymbol{v}_{2}, 0\right)$ give the restrictions

$$
u_{11} u_{22}-u_{12}^{2}=\eta_{1} \eta_{2}, u_{11}^{2}+u_{22}^{2}+2 u_{12}^{2}+2\left|u_{12}\left(u_{11}+u_{22}\right)\right| \leq \eta_{1}^{2}+\eta_{2}^{2} .
$$

The components $\left(u_{11}, u_{12}\right)$ of tensors satisfying (3.6-3.7) are represented in Fig. 1 (left) in the case $\eta_{2} / \eta_{1}=0.5$. For latter reference, observe that there 
is an upper limit $u_{12}^{0}$ on the shear component $\left|u_{12}\right|$ of such tensors. It can be calculated from (3.7) that

$$
u_{12}^{0}=\frac{\left(\eta_{1}-\eta_{2}\right)^{2}}{2 \sqrt{2\left(\eta_{1}^{2}+\eta_{2}^{2}\right)}} .
$$

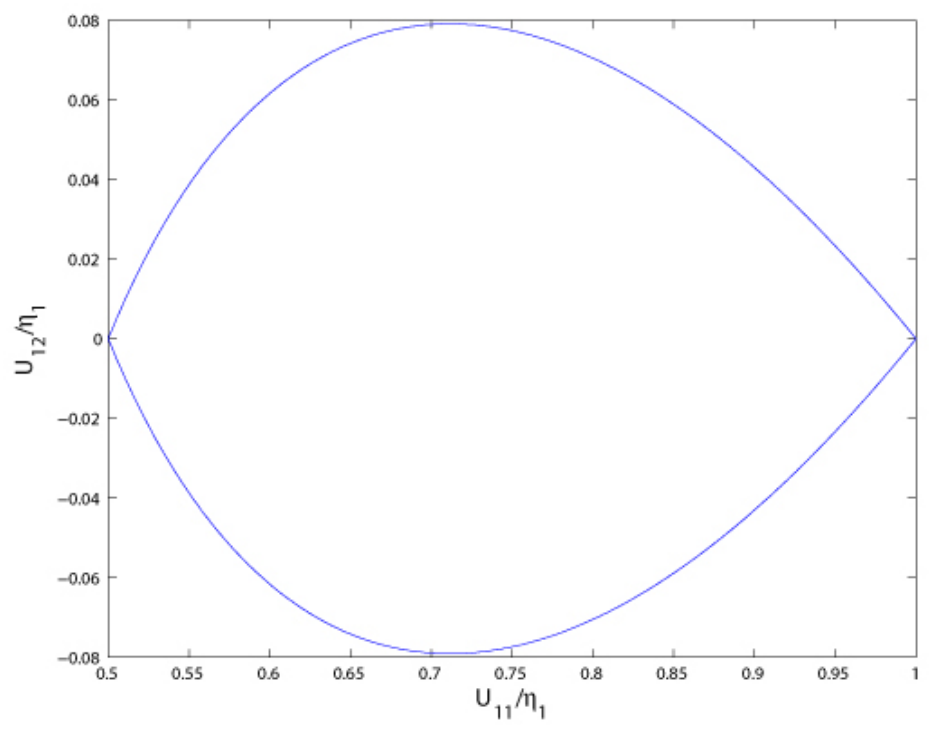

Figure 1: representation of the set $\mathcal{U}^{0}$ in the two-well problem $\left(\eta_{2} / \eta_{1}=0.5\right)$.

The arguments used so far only ensure that $\mathcal{U}^{0}$ is included in the set defined by (3.6-3.7). The reverse inclusion is also true: for any given $\overline{\boldsymbol{U}}$ satisfying (3.6-3.7), Ball and James (1992) explicitly constructed a laminate microstructure that realizes $\overline{\boldsymbol{U}}$. More precisely, we have the following theorem:

Theorem 3. (Ball and James, 1992) For the two-well problem (3.1), any deformation gradient $\overline{\boldsymbol{F}}$ in $Q \mathcal{K}$ is realized by a second-rank laminate. There is not unicity of the gradient Young measure, except when $\overline{\boldsymbol{F}}$ can be realized by a first-rank laminate.

The conclusion is that the set defined by (3.6-3.7) gives the exact expression of $\mathcal{U}^{0}$. Referring to Theorem 3, the deformation gradients $\overline{\boldsymbol{F}}$ that can be realized by first-rank laminates take the form $\lambda \boldsymbol{R} \boldsymbol{U}_{2}+(1-\lambda) \boldsymbol{U}_{1}$ where $\boldsymbol{R}$ 
is solution of the twinning equation (3.2) and $0 \leq \lambda \leq 1$. Such deformation gradients correspond to points on the boundary of the domain represented in Fig. 1.

Using the solution of the two-well problem, the following property is proved in Appendix A and will be useful in the next Sections:

Theorem 4. Assume $\left(\boldsymbol{U}_{1}, \boldsymbol{U}_{2}\right)$ satisfy (3.1) and take $\overline{\boldsymbol{F}}$ in $Q\left(S O(3) \boldsymbol{U}_{1} \cup\right.$ $\left.S O(3) \boldsymbol{U}_{2}\right)$. Then $\operatorname{Ker}\left(\boldsymbol{U}_{1}^{2}-\boldsymbol{U}_{2}^{2}\right)$ is of dimension 1, and $\|\overline{\boldsymbol{F}} \cdot \boldsymbol{v}\|=\left\|\boldsymbol{U}_{1} . \boldsymbol{v}\right\|$ for any $\boldsymbol{v} \in \operatorname{Ker}\left(\boldsymbol{U}_{1}^{2}-\boldsymbol{U}_{2}^{2}\right)$. Similarly, $\operatorname{Ker}\left(\boldsymbol{U}_{1}^{*, 2}-\boldsymbol{U}_{2}^{*, 2}\right)$ is of dimension 1, and $\left\|\overline{\boldsymbol{F}}^{*} \cdot \boldsymbol{w}\right\|=\left\|\boldsymbol{U}_{1}^{*} \cdot \boldsymbol{w}\right\|$ for any $\boldsymbol{w} \in \operatorname{Ker}\left(\boldsymbol{U}_{1}^{*, 2}-\boldsymbol{U}_{2}^{*, 2}\right)$.

Let us address some comments on that theorem. For $\left(\boldsymbol{U}_{1}, \boldsymbol{U}_{2}\right)$ satisfying (3.1), the fact that $\operatorname{Ker}\left(\boldsymbol{U}_{1}^{2}-\boldsymbol{U}_{2}^{2}\right)$ is of dimension 1 means that there exists a non zero vector $\boldsymbol{v}$ (unique up to a multiplicative constant) such that $\left\|\boldsymbol{U}_{1} \cdot \boldsymbol{v}\right\|=\left\|\boldsymbol{U}_{2} \cdot \boldsymbol{v}\right\|$. In such condition, any $\overline{\boldsymbol{F}}$ in $\mathrm{SO}(3) \boldsymbol{U}_{1} \cup \mathrm{SO}(3) \boldsymbol{U}_{2}$ satisfies $\|\overline{\boldsymbol{F}} \cdot \boldsymbol{v}\|=\left\|\boldsymbol{U}_{1} . \boldsymbol{v}\right\|$. Theorem 4 shows that the equality $\|\overline{\boldsymbol{F}} \cdot \boldsymbol{v}\|=\left\|\boldsymbol{U}_{1} \cdot \boldsymbol{v}\right\|$ is satisfied not only for $\overline{\boldsymbol{F}}$ in $\mathrm{SO}(3) \boldsymbol{U}_{1} \cup \mathrm{SO}(3) \boldsymbol{U}_{2}$, but also for $\overline{\boldsymbol{F}}$ in the quasiconvex hull $Q\left(\mathrm{SO}(3) \boldsymbol{U}_{1} \cup \mathrm{SO}(3) \boldsymbol{U}_{2}\right)$. Similar properties hold for adjugates: there exists a non zero vector $\boldsymbol{w}$ (unique up to a multiplicative constant) such that $\left\|\boldsymbol{U}_{1}^{*} \cdot \boldsymbol{w}\right\|=\left\|\boldsymbol{U}_{2}^{*} \cdot \boldsymbol{w}\right\|=\left\|\overline{\boldsymbol{F}}^{*} \cdot \boldsymbol{w}\right\|$ for any $\overline{\boldsymbol{F}}$ in $Q\left(\operatorname{SO}(3) \boldsymbol{U}_{1} \cup \operatorname{SO}(3) \boldsymbol{U}_{2}\right)$.

As a final remark on the two-well problem, we note that the bound $P_{0} \mathcal{K}$ in (2.25) happens to give the exact value of $\mathcal{K}_{0}$, and that rank-1 tensors $(\boldsymbol{\tau}, \boldsymbol{a})$ reach the supremum in (2.25). Those properties are associated with the two-dimensional nature of the two-well problem: as will be illustrated in the next Section, the inclusions $Q \mathcal{K} \subset P \mathcal{K} \subset P_{0} \mathcal{K}$ are generally strict, and rank-1 tensors $(\boldsymbol{\tau}, \boldsymbol{a})$ are not necessarily optimal in (2.24) or (2.25).

\section{A three-well problem}

In this section we study the three-well problem $\mathcal{K}=\bigcup_{r=1}^{3} \mathrm{SO}(3) \boldsymbol{U}_{r}$ where $\boldsymbol{U}_{1}, \boldsymbol{U}_{2}, \boldsymbol{U}_{3}$ admit the matrix representations

$$
\boldsymbol{U}_{1}=\left(\begin{array}{ccc}
\mu_{2} & 0 & 0 \\
0 & \mu_{1} & 0 \\
0 & 0 & \mu_{1}
\end{array}\right), \boldsymbol{U}_{2}=\left(\begin{array}{ccc}
\eta_{1} & 0 & 0 \\
0 & \eta_{2} & 0 \\
0 & 0 & \eta_{1}
\end{array}\right), \boldsymbol{U}_{3}=\left(\begin{array}{ccc}
\eta_{1} & 0 & 0 \\
0 & \eta_{1} & 0 \\
0 & 0 & \eta_{2}
\end{array}\right)
$$

in the reference basis $\left(\boldsymbol{v}_{1}, \boldsymbol{v}_{2}, \boldsymbol{v}_{3}\right)$. The parameters $\left(\eta_{1}, \eta_{2}, \mu_{1}, \mu_{2}\right)$ are positive, distinct and assumed to satisfy $\eta_{1}^{2} \eta_{2}=\mu_{1}^{2} \mu_{2}$, so that $\boldsymbol{U}_{1}, \boldsymbol{U}_{2}, \boldsymbol{U}_{3}$ have the 
same determinant. For any second-order tensor $\boldsymbol{M}$, the scalar $\boldsymbol{v}_{i} \cdot \boldsymbol{M} \cdot \boldsymbol{v}_{j}$ is denoted by $M_{i j}$. We start by studying in detail the case $\mu_{1}=\eta_{1}$ and hence $\mu_{2}=\eta_{2}$, which corresponds to the cubic to tetragonal transformation. We derive an explicit upper bound on $Q \mathcal{K}$, investigate its structure and compare it with lower bounds constructed by sequential lamination techniques. We then study the situation $\mu_{1} \neq \eta_{1}$, in which case the three wells are not all pairwise rank- 1 connected. The cases $\mu_{1} \in\left[\eta_{2}, \eta_{1}\right]$ and $\mu_{1} \notin\left[\eta_{2}, \eta_{1}\right]$ (with $\eta_{1}$ assumed to be larger than $\eta_{2}$ ) are studied separately as they lead to different structures for the set $Q \mathcal{K}$.

\subsection{Case $\mu_{1}=\eta_{1}$ : cubic to tetragonal transformation}

We first assume that $\eta_{1}>\eta_{2}$. Let $\{i, j, k\}$ be a permutation of $\{1,2,3\}$. Using the relation (2.19) and (2.30) with $\left(\boldsymbol{v}, \boldsymbol{v}^{\prime}\right)=\left(\boldsymbol{v}_{k}, 0\right),\left(0, \boldsymbol{v}_{k}\right)$, we obtain that any $\overline{\boldsymbol{F}}$ in $Q \mathcal{K}$ satisfies the condition $\operatorname{det} \overline{\boldsymbol{F}}=\eta_{1}^{2} \eta_{2}$ and

$$
\left\|\overline{\boldsymbol{F}} \cdot \boldsymbol{v}_{k}\right\| \leq \eta_{1}-\theta_{k}\left(\eta_{1}-\eta_{2}\right),\left\|\overline{\boldsymbol{F}}^{*} \cdot \boldsymbol{v}_{k}\right\| \leq \eta_{1} \eta_{2}+\theta_{k} \eta_{1}\left(\eta_{1}-\eta_{2}\right)
$$

for some $\boldsymbol{\theta}$ in $\mathcal{T}_{3}$. Since $\eta_{1}>\eta_{2}$, the inequality (4.2) can be rewritten as

$$
\frac{\left\|\overline{\boldsymbol{F}}^{*} \cdot \boldsymbol{v}_{k}\right\|-\eta_{1} \eta_{2}}{\eta_{1}\left(\eta_{1}-\eta_{2}\right)} \leq \theta_{k} \leq \frac{\eta_{1}-\left\|\overline{\boldsymbol{F}} \cdot \boldsymbol{v}_{k}\right\|}{\eta_{1}-\eta_{2}} .
$$

Let $\epsilon_{k} \in\{-1,+1\}$. Using now (2.30) with $\left(\boldsymbol{v}, \boldsymbol{v}^{\prime}\right)=\left(\boldsymbol{v}_{i}+\epsilon_{k} \boldsymbol{v}_{j}, 0\right)$ and $\left(\boldsymbol{v}, \boldsymbol{v}^{\prime}\right)=\left(0, \boldsymbol{v}_{i}+\epsilon_{k} \boldsymbol{v}_{j}\right)$ yields

$$
\frac{\left\|\overline{\boldsymbol{F}} \cdot\left(\boldsymbol{v}_{i}+\epsilon_{k} \boldsymbol{v}_{j}\right)\right\|-\sqrt{\eta_{1}^{2}+\eta_{2}^{2}}}{\sqrt{2} \eta_{1}-\sqrt{\eta_{1}^{2}+\eta_{2}^{2}}} \leq \theta_{k} \leq \frac{\eta_{1} \sqrt{\eta_{1}^{2}+\eta_{2}^{2}}-\left\|\overline{\boldsymbol{F}}^{*} \cdot\left(\boldsymbol{v}_{i}+\epsilon_{k} \boldsymbol{v}_{j}\right)\right\|}{\eta_{1}\left(\sqrt{\eta_{1}^{2}+\eta_{2}^{2}}-\sqrt{2} \eta_{2}\right)} .
$$

Combining (4.3) and (4.4), we obtain

$$
A_{k}(\overline{\boldsymbol{F}}) \leq \theta_{k} \leq B_{k}(\overline{\boldsymbol{F}})
$$

with

$$
\begin{aligned}
& A_{k}(\overline{\boldsymbol{F}})=\max \left(\frac{\left\|\overline{\boldsymbol{F}}^{*} \cdot \boldsymbol{v}_{k}\right\|-\eta_{1} \eta_{2}}{\eta_{1}\left(\eta_{1}-\eta_{2}\right)}, \frac{\left\|\overline{\boldsymbol{F}} \cdot\left(\boldsymbol{v}_{i}+\epsilon_{k} \boldsymbol{v}_{j}\right)\right\|-\sqrt{\eta_{1}^{2}+\eta_{2}^{2}}}{\sqrt{2} \eta_{1}-\sqrt{\eta_{1}^{2}+\eta_{2}^{2}}}\right) \\
& B_{k}(\overline{\boldsymbol{F}})=\min \left(\frac{\eta_{1}-\left\|\overline{\boldsymbol{F}} \cdot \boldsymbol{v}_{k}\right\|}{\eta_{1}-\eta_{2}}, \frac{\eta_{1} \sqrt{\eta_{1}^{2}+\eta_{2}^{2}}-\left\|\overline{\boldsymbol{F}}^{*} \cdot\left(\boldsymbol{v}_{i}+\epsilon_{k} \boldsymbol{v}_{j}\right)\right\|}{\eta_{1}\left(\sqrt{\eta_{1}^{2}+\eta_{2}^{2}}-\sqrt{2} \eta_{2}\right)}\right) .
\end{aligned}
$$


In those definitions, the maximum and the minimum are taken over $\epsilon_{k} \in$ $\{-1,1\}$ and over $\{i, j\}$ such that $\{i, j, k\}$ is a permutation of $\{1,2,3\}$. The restrictions (4.5) correspond to the maximization of (2.24) over tensors $(\boldsymbol{\tau}, \boldsymbol{a})$ assuming one of the following forms:

$$
\left(\left(\boldsymbol{v}_{i}+\epsilon_{k} \boldsymbol{v}_{j}\right) \otimes \boldsymbol{w}, 0\right),\left(0,\left(\boldsymbol{v}_{i}+\epsilon_{k} \boldsymbol{v}_{j}\right) \otimes \boldsymbol{w}\right),\left(\boldsymbol{v}_{k} \otimes \boldsymbol{w}, 0\right),\left(0, \boldsymbol{v}_{k} \otimes \boldsymbol{w}\right)
$$

where $\boldsymbol{w}$ is an arbitrary vector. The restrictions (4.5) can be improved by maximizing (2.24) with respect to tensors $(\boldsymbol{\tau}, \boldsymbol{a})$ of the form

$$
\left(\sum_{i=1}^{3} \tau_{i} \boldsymbol{v}_{i} \otimes \boldsymbol{v}_{i} . \boldsymbol{R}, \sum_{i=1}^{3} a_{i} \boldsymbol{v}_{i} \otimes \boldsymbol{v}_{i} . \boldsymbol{R}\right)
$$

where $\boldsymbol{R}$ is a rotation and $\left(\tau_{i}, a_{i}\right)_{1 \leq i \leq 3}$ are arbitrary scalars. In such case, the tensor $\boldsymbol{U}_{r} . \boldsymbol{\tau}+\boldsymbol{U}_{r}^{*} . \boldsymbol{a}$ in (2.24) can be written as $\sum_{i} M_{i} \boldsymbol{v}_{i} \otimes \boldsymbol{v}_{i} . \boldsymbol{R}$. Note from the expression (2.22) that $\Phi\left(\sum_{i} M_{i} \boldsymbol{v}_{i} \otimes \boldsymbol{v}_{i} . \boldsymbol{R}\right)=f\left(M_{1}, M_{2}, M_{3}\right)$. Substituting (4.8) in the inequality (2.24) shows that any $\overline{\boldsymbol{F}}$ in $Q \mathcal{K}$ satisfies

$$
\begin{aligned}
& 0 \geq \sup _{\boldsymbol{R} \in \mathrm{SO}(3)} \sup _{a_{i}, \tau_{i}}\left\{\overline{\boldsymbol{F}}:\left(\sum_{i=1}^{3} \tau_{i} \boldsymbol{v}_{i} \otimes \boldsymbol{v}_{i} . \boldsymbol{R}\right)+\overline{\boldsymbol{F}}^{*}:\left(\sum_{i=1}^{3} a_{i} \boldsymbol{v}_{i} \otimes \boldsymbol{v}_{i} . \boldsymbol{R}\right)\right. \\
& \left.-\theta_{1} f\left(y_{1}, x_{2}, x_{3}\right)-\theta_{2} f\left(x_{1}, y_{2}, x_{3}\right)-\theta_{3} f\left(x_{1}, x_{2}, y_{3}\right)\right\}
\end{aligned}
$$

where

$$
x_{i}=n_{1} \tau_{i}+\eta_{1} \eta_{2} a_{i}, y_{i}=\eta_{2} \tau_{i}+\eta_{1}^{2} a_{i}
$$

for $i=1,2,3$. Using (4.10) to express $\left(\tau_{i}, a_{i}\right)$ as a function of $\left(x_{i}, y_{i}\right)$, the inequality (4.9) can be rewritten as

$$
\begin{aligned}
0 \geq & \sup _{\boldsymbol{R} \in \operatorname{SO}(3)} \sup _{x_{i}, y_{i}}\left\{\sum_{i=1}^{3}\left(l_{i}(\boldsymbol{R} . \overline{\boldsymbol{F}}) x_{i}+m_{i}(\boldsymbol{R} . \overline{\boldsymbol{F}}) y_{i}\right)\right. \\
& \left.-\theta_{1} f\left(y_{1}, x_{2}, x_{3}\right)-\theta_{2} f\left(x_{1}, y_{2}, x_{3}\right)-\theta_{3} f\left(x_{1}, x_{2}, y_{3}\right)\right\}
\end{aligned}
$$

where the operators $l_{i}$ and $m_{i}$ are defined for any second-order tensor $\boldsymbol{M}$ by

$$
l_{i}(\boldsymbol{M})=\frac{\eta_{1}^{2} M_{i i}-\eta_{2} M_{i i}^{*}}{\eta_{1}\left(\eta_{1}^{2}-\eta_{2}^{2}\right)}, m_{i}(\boldsymbol{M})=\frac{-\eta_{2} M_{i i}+M_{i i}^{*}}{\eta_{1}^{2}-\eta_{2}^{2}}
$$

Considering $\overline{\boldsymbol{F}}$ and $\boldsymbol{R}$ as fixed, the right-hand side of (4.11) can be maximized with respect to the arbitrary sextuplet $\left(x_{i}, y_{i}\right)_{1 \leq i \leq 3}$. From the definition (2.23) of $f$, it can be noticed that $\left(x_{i}, y_{i}\right) \mapsto f\left(y_{1}, x_{2}, x_{3}\right)$ is piecewise 
linear and depends on the ordering of $\left\{0, y_{1}, x_{2}, x_{3}\right\}$. Similar remarks prevail for $f\left(x_{1}, y_{2}, y_{3}\right)$ and $f\left(x_{1}, x_{2}, y_{3}\right)$. The maximization of (4.11) with respect to $\left(x_{i}, y_{i}\right)_{1 \leq i \leq 3}$ thus amounts to solve a series of constrained linear optimization problems, obtained by restricting the optimization of (4.11) to values $\left(x_{i}, y_{i}\right)_{1 \leq i \leq 3}$ compatible with prescribed orderings of $\left\{0, x_{1}, x_{2}, x_{3}, y_{1}, y_{2}, y_{3}\right\}$. Carrying out these calculations shows that there is a total of 26 optimal sex-

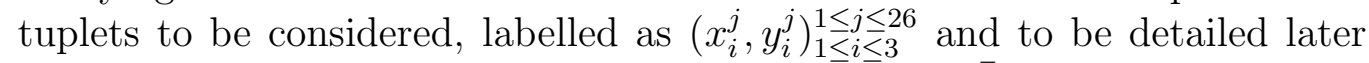
on. That family is optimal in the sense that for any $\overline{\boldsymbol{F}}$ and $\boldsymbol{R}$, there exists $1 \leq j \leq 26$ such that the supremum over $\left(x_{i}, y_{i}\right)$ in $(4.11)$ is reached for $\left(x_{i}, y_{i}\right)_{1 \leq i \leq 3}=\left(x_{i}^{j}, y_{i}^{j}\right)_{1 \leq i \leq 3}$. The inequality (4.11) is thus equivalent to

$$
0 \geq \quad \begin{aligned}
& \sup _{\boldsymbol{R} \in \mathrm{SO}(3)} \max _{1 \leq j \leq 26}\left\{\sum_{i=1}^{3}\left(l_{i}(\boldsymbol{R} . \overline{\boldsymbol{F}}) x_{i}^{j}+m_{i}(\boldsymbol{R} . \overline{\boldsymbol{F}}) y_{i}^{j}\right)\right. \\
& \left.-\theta_{1} f\left(y_{1}^{j}, x_{2}^{j}, x_{3}^{j}\right)-\theta_{2} f\left(x_{1}^{j}, y_{2}^{j}, x_{3}^{j}\right)-\theta_{3} f\left(x_{1}^{j}, x_{2}^{j}, y_{3}^{j}\right)\right\} .
\end{aligned}
$$

For any $1 \leq j \leq 26$, define $\boldsymbol{\tau}^{j}=\sum_{i} \tau_{i}^{j} \boldsymbol{v}_{i} \otimes \boldsymbol{v}_{i}, \boldsymbol{a}^{j}=\sum_{i} a_{i}^{j} \boldsymbol{v}_{i} \otimes \boldsymbol{v}_{i}$ where $\left(\tau_{i}^{j}, a_{i}^{j}\right)$ is related to $\left(x_{i}^{j}, y_{i}^{j}\right)$ via (4.10). Interchanging the supremum and the maximum, the inequality (4.13) can be rewritten as

$$
\begin{aligned}
0 \geq & \max _{1 \leq j \leq 26} \sup _{\boldsymbol{R} \in \mathrm{SO}(3)}\left\{\boldsymbol{R} \overline{\boldsymbol{F}}: \boldsymbol{\tau}^{j}+\boldsymbol{R} \overline{\boldsymbol{F}}^{*}: \boldsymbol{a}^{j}\right. \\
& \left.-\theta_{1} f\left(y_{1}^{j}, x_{2}^{j}, x_{3}^{j}\right)-\theta_{2} f\left(x_{1}^{j}, y_{2}^{j}, x_{3}^{j}\right)-\theta_{3} f\left(x_{1}^{j}, x_{2}^{j}, y_{3}^{j}\right)\right\} .
\end{aligned}
$$

In this inequality, the maximization problem over $\boldsymbol{R}$ can be solved with the help of the function $\Phi$ introduced in (2.21). We get

$$
0 \geq \Phi\left(\overline{\boldsymbol{F}} \cdot \boldsymbol{\tau}^{j}+\overline{\boldsymbol{F}}^{*} \cdot \boldsymbol{a}^{j}\right)-\theta_{1} f\left(y_{1}^{j}, x_{2}^{j}, x_{3}^{j}\right)-\theta_{2} f\left(x_{1}^{j}, y_{2}^{j}, x_{3}^{j}\right)-\theta_{3} f\left(x_{1}^{j}, x_{2}^{j}, y_{3}^{j}\right)
$$

for $1 \leq j \leq 26$.

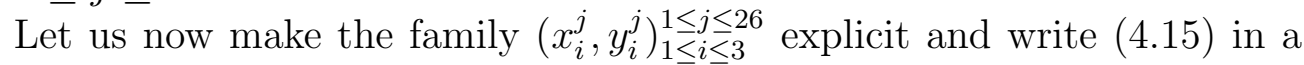
more convenient form. That family can be broken down into three groups. The first group, labeled as $\left(x_{i}^{j}, y_{i}^{j}\right)_{1 \leq i \leq 3}^{1 \leq j \leq 6}$, corresponds to sextuplets with one component equal to 1 , and all the others equal to 0 . In the case where $x_{k}^{j}=1$ for a certain $k$, the corresponding tensors $\left(\boldsymbol{\tau}^{j}, \boldsymbol{a}^{j}\right)$ are given by

$$
\boldsymbol{\tau}^{j}=\frac{\eta_{1}}{\eta_{1}^{2}-\eta_{2}^{2}} \boldsymbol{v}_{k} \otimes \boldsymbol{v}_{k}, \boldsymbol{a}^{j}=-\frac{\eta_{2}}{\eta_{1}\left(\eta_{1}^{2}-\eta_{2}^{2}\right)} \boldsymbol{v}_{k} \otimes \boldsymbol{v}_{k}
$$


and the inequality $(4.15)$ can be written as $0 \geq \phi_{k}(\overline{\boldsymbol{F}})-\left(1-\theta_{k}\right)$ where

$$
\begin{aligned}
\phi_{k}(\overline{\boldsymbol{F}}) & =\frac{1}{\eta_{1}\left(\eta_{1}^{2}-\eta_{2}^{2}\right)} \Phi\left(\left(\eta_{1}^{2} \overline{\boldsymbol{F}}-\eta_{2} \overline{\boldsymbol{F}}^{*}\right) \cdot \boldsymbol{v}_{k} \otimes \boldsymbol{v}_{k}\right) \\
& =\frac{1}{\eta_{1}\left(\eta_{1}^{2}-\eta_{2}^{2}\right)}\left\|\eta_{1}^{2} \overline{\boldsymbol{F}} \cdot \boldsymbol{v}_{k}-\eta_{2} \overline{\boldsymbol{F}}^{*} \cdot \boldsymbol{v}_{k}\right\| .
\end{aligned}
$$

In the case where $y_{k}^{j}=1$ for a certain $k$, we arrive at $0 \geq \psi_{k}(\overline{\boldsymbol{F}})-\theta_{k}$ where $\psi_{k}$ is defined by

$$
\psi_{k}(\overline{\boldsymbol{F}})=\frac{1}{\eta_{1}^{2}-\eta_{2}^{2}}\left\|\eta_{2} \overline{\boldsymbol{F}} \cdot \boldsymbol{v}_{k}-\overline{\boldsymbol{F}}^{*} \cdot \boldsymbol{v}_{k}\right\|
$$

The second group in the family $\left(x_{i}^{j}, y_{i}^{j}\right)_{1 \leq i \leq 3}^{1 \leq j \leq 26}$ consists of sextuplets of the form

$$
\begin{aligned}
& x_{i_{1}}=\epsilon \quad, \quad x_{i_{2}}=\epsilon^{\prime} \quad, \quad x_{i_{3}}=0, \\
& y_{i_{1}}=0 \quad, \quad y_{i_{2}}=0 \quad, \quad y_{i_{3}}=-\epsilon \epsilon^{\prime} \text {, }
\end{aligned}
$$

where $\epsilon= \pm 1, \epsilon^{\prime}= \pm 1$ and $\left\{i_{1}, i_{2}, i_{3}\right\}$ is a permutation of $\{1,2,3\}$. There are

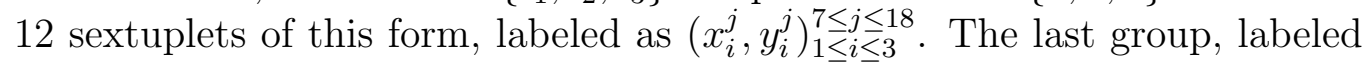
as $\left(x_{i}^{j}, y_{i}^{j}\right)_{1 \leq i \leq 3}^{19 \leq j \leq 26}$, is formed by the eight sextuplets of the form

$$
x_{i}=\epsilon_{i}, y_{i}=-\frac{\epsilon_{1} \epsilon_{2} \epsilon_{3}}{\epsilon_{i}}
$$

where $\epsilon_{i}= \pm 1$. Observe from the expression (4.19) and (4.20) that $f\left(y_{1}^{j}, x_{2}^{j}, x_{3}^{j}\right)=$ $f\left(x_{1}^{j}, y_{2}^{j}, x_{3}^{j}\right)=f\left(x_{1}^{j}, x_{2}^{j}, y_{3}^{j}\right)=1$ for $j>6$, so that the inequality (4.15) becomes $\Phi\left(\overline{\boldsymbol{F}} \cdot \boldsymbol{\tau}^{j}+\overline{\boldsymbol{F}}^{*} \cdot \boldsymbol{a}^{j}\right) \leq 1$. The tensors $\left(\boldsymbol{\tau}^{j}, \boldsymbol{a}^{j}\right)$ corresponding to (4.19) are

$$
\begin{array}{ll}
\boldsymbol{\tau}^{j}= & \frac{1}{\eta_{1}^{2}-\eta_{2}^{2}} \quad\left(\epsilon \eta_{1} \boldsymbol{v}_{i_{1}} \otimes \boldsymbol{v}_{i_{1}}+\epsilon^{\prime} \eta_{1} \boldsymbol{v}_{i_{2}} \otimes \boldsymbol{v}_{i_{2}}+\epsilon \epsilon^{\prime} \eta_{2} \boldsymbol{v}_{i_{3}} \otimes \boldsymbol{v}_{i_{3}}\right), \\
\boldsymbol{a}^{j}=-\frac{1}{\eta_{1}\left(\eta_{1}^{2}-\eta_{2}^{2}\right)} & \left(\epsilon \eta_{2} \boldsymbol{v}_{i_{1}} \otimes \boldsymbol{v}_{i_{1}}+\epsilon^{\prime} \eta_{2} \boldsymbol{v}_{i_{2}} \otimes \boldsymbol{v}_{i_{2}}+\epsilon \epsilon^{\prime} \eta_{1} \boldsymbol{v}_{i_{3}} \otimes \boldsymbol{v}_{i_{3}}\right),
\end{array}
$$

whereas the tensors $\left(\boldsymbol{\tau}^{j}, \boldsymbol{a}^{j}\right)$ corresponding to (4.20) are

$$
\begin{aligned}
\boldsymbol{\tau}^{j} & =\frac{1}{\eta_{1}^{2}-\eta_{2}^{2}} \sum_{i=1}^{3} \epsilon_{i}\left(\eta_{1}+\epsilon_{1} \epsilon_{2} \epsilon_{3} \eta_{2}\right) \boldsymbol{v}_{i} \otimes \boldsymbol{v}_{i}, \\
\boldsymbol{a}^{j} & =-\frac{1}{\eta_{1}\left(\eta_{1}^{2}-\eta_{2}^{2}\right)} \sum_{i=1}^{3} \epsilon_{i}\left(\eta_{2}+\epsilon_{1} \epsilon_{2} \epsilon_{3} \eta_{1}\right) \boldsymbol{v}_{i} \otimes \boldsymbol{v}_{i} .
\end{aligned}
$$


In summary, (4.15) can be explicited as

$$
\begin{gathered}
\psi_{k}(\overline{\boldsymbol{F}}) \leq \theta_{k} \leq 1-\phi_{k}(\overline{\boldsymbol{F}}) \text { for } 1 \leq k \leq 3, \\
\Phi\left(\overline{\boldsymbol{F}} \cdot \boldsymbol{\tau}^{j}+\overline{\boldsymbol{F}}^{*} \cdot \boldsymbol{a}^{j}\right) \leq 1 \text { for } 7 \leq j \leq 26 .
\end{gathered}
$$

Combining this equation with (4.5), we obtain that

$$
A_{k}^{\prime}(\overline{\boldsymbol{F}}) \leq \theta_{k} \leq B_{k}^{\prime}(\overline{\boldsymbol{F}})
$$

where

$$
A_{k}^{\prime}(\overline{\boldsymbol{F}})=\max \left(A_{k}(\overline{\boldsymbol{F}}), \psi_{k}(\overline{\boldsymbol{F}})\right), B_{k}^{\prime}(\overline{\boldsymbol{F}})=\min \left(B_{k}(\overline{\boldsymbol{F}}), 1-\phi_{k}(\overline{\boldsymbol{F}})\right) .
$$

Using the fact that any $\boldsymbol{\theta}$ in $\mathcal{T}_{3}$ satisfies $\theta_{i} \geq 0$ and $\sum_{i} \theta_{i}=1$, we find that any $\overline{\boldsymbol{F}}$ in $Q \mathcal{K}$ satisfies

$$
\begin{gathered}
A_{k}^{\prime}(\overline{\boldsymbol{F}}) \leq B_{k}^{\prime}(\overline{\boldsymbol{F}}) \text { for } k=1,2,3 \\
\sum_{k=1}^{3} A_{k}^{\prime}(\overline{\boldsymbol{F}}) \leq 1 \leq \sum_{k=1}^{3} B_{k}^{\prime}(\overline{\boldsymbol{F}})
\end{gathered}
$$

and

$$
\max _{7 \leq j \leq 26} \Phi\left(\overline{\boldsymbol{F}} \cdot \boldsymbol{\tau}^{j}+\overline{\boldsymbol{F}}^{*} \cdot \boldsymbol{a}^{j}\right) \leq 1 .
$$

The expressions (4.25)-(4.26) are the result of optimizing (2.24) with respect to tensors $(\boldsymbol{\tau}, \boldsymbol{a})$ of the form (4.7) and (4.8). The bound given by (4.25)(4.26) is still denoted by $P \mathcal{K}$, although - contrary to the two-well problem it is not ensured that tensors of the form (4.7) or (4.8) reach the supremum in (2.24). The bound $P_{0} \mathcal{K}$ obtained by optimizing (2.25) with respect to the same class of tensors is given by the restrictions (4.26) along with the following additional requirement:

$$
A_{k}^{\prime}(\overline{\boldsymbol{F}}) \leq 1,0 \leq B_{k}^{\prime}(\overline{\boldsymbol{F}}) \text { for } k=1,2,3 .
$$

Remark 1: We have not been able to find an example of deformation gradient $\overline{\boldsymbol{F}}$ which satisfies (4.25) but not (4.26), although no attempt has been made to rigorously prove that such an example does not exist. This might suggest that the restriction (4.26) is redundant and can be omitted without losing any information. 
Remark 2: We have assumed so far that $\eta_{1}>\eta_{2}$. When $\eta_{2}>\eta_{1}$, we obtain that $P \mathcal{K}$ is defined by inequalities analog to (4.25) and (4.26) except that $A_{k}(\overline{\boldsymbol{F}}), B_{k}(\overline{\boldsymbol{F}}), \phi_{k}(\overline{\boldsymbol{F}}), \psi_{k}(\overline{\boldsymbol{F}})$ are defined by

$$
\begin{aligned}
A_{k}(\overline{\boldsymbol{F}}) & =\min \left(\frac{\eta_{1}-\left\|\overline{\boldsymbol{F}} \cdot \boldsymbol{v}_{k}\right\|}{\eta_{1}-\eta_{2}}, \frac{\eta_{1} \sqrt{\eta_{1}^{2}+\eta_{2}^{2}}-\left\|\overline{\boldsymbol{F}}^{*} \cdot\left(\boldsymbol{v}_{i}+\epsilon_{k} \boldsymbol{v}_{j}\right)\right\|}{\eta_{1}\left(\sqrt{\eta_{1}^{2}+\eta_{2}^{2}}-\sqrt{2} \eta_{2}\right)}\right), \\
B_{k}(\overline{\boldsymbol{F}}) & =\max \left(\frac{\left\|\overline{\boldsymbol{F}}^{*} \cdot \boldsymbol{v}_{k}\right\|-\eta_{1} \eta_{2}}{\eta_{1}\left(\eta_{1}-\eta_{2}\right)}, \frac{\left\|\overline{\boldsymbol{F}} \cdot\left(\boldsymbol{v}_{i}+\epsilon_{k} \boldsymbol{v}_{j}\right)\right\|-\sqrt{\eta_{1}^{2}+\eta_{2}^{2}}}{\sqrt{2} \eta_{1}-\sqrt{\eta_{1}^{2}+\eta_{2}^{2}}}\right), \\
\phi_{k}(\overline{\boldsymbol{F}}) & =\frac{1}{\eta_{1}\left(\eta_{2}^{2}-\eta_{1}^{2}\right)}\left\|\eta_{1}^{2} \overline{\boldsymbol{F}} \cdot \boldsymbol{v}_{k}-\eta_{2} \overline{\boldsymbol{F}}^{*} \cdot \boldsymbol{v}_{k}\right\|, \\
\psi_{k}(\overline{\boldsymbol{F}}) & =\frac{1}{\eta_{2}^{2}-\eta_{1}^{2}}\left\|\eta_{2} \overline{\boldsymbol{F}} \cdot \boldsymbol{v}_{k}-\overline{\boldsymbol{F}}^{*} \cdot \boldsymbol{v}_{k}\right\| .
\end{aligned}
$$

In the rest of this section, we compare the upper bound $P \mathcal{K}$ in (4.25) with some lower bounds obtained from considerations of laminate microstructures. This comparison is supported by graphical representations of the bounding sets considered. For that purpose, it is necessary to consider cross-sections obtained by assuming a certain form of $\overline{\boldsymbol{F}}$. Since $Q \mathcal{K}$ and $P \mathcal{K}$ are frame indifferent, it is sufficient to consider symmetric positive tensors. For any given $u_{12}^{*}$, we consider the set $\mathcal{U}\left(u_{12}^{*}\right)$ of deformation gradients in $Q \mathcal{K}$ with a matrix representation of the form

$$
\left(\begin{array}{ccc}
u_{11} & u_{12}^{*} & 0 \\
u_{12}^{*} & u_{22} & 0 \\
0 & 0 & u_{33}
\end{array}\right)
$$

Because of the condition $\operatorname{det} \boldsymbol{U}=\eta_{1}^{2} \eta_{2}$, any tensor $\boldsymbol{U}$ in $\mathcal{U}\left(u_{12}^{*}\right)$ is determined by its components $u_{11}$ and $u_{22}$. Therefore $\mathcal{U}\left(u_{12}^{*}\right)$ can be identified with its projection on the plane $\left(u_{11}, u_{22}\right)$, which will be done in the following.

Using the results collected in Section 3 for the two-well problem, we first observe that $Q \mathcal{K}$ contains all the tensors assuming one of the following forms:

$$
\left(\begin{array}{ccc}
\eta_{1} & 0 & 0 \\
0 & a & c \\
0 & c & b
\end{array}\right),\left(\begin{array}{ccc}
a & 0 & c \\
0 & \eta_{1} & 0 \\
c & 0 & b
\end{array}\right)
$$

where $a, b, c$ are submitted to the restrictions (3.7). In particular, it can be seen from (4.30) that $Q \mathcal{K}$ contains the tensors $\boldsymbol{W}_{i}(\lambda)$ defined by

$$
\boldsymbol{W}_{1}(\lambda)=\operatorname{diag}\left(\eta_{1}, \lambda, \frac{\eta_{1} \eta_{2}}{\lambda}\right), \boldsymbol{W}_{2}(\lambda)=\operatorname{diag}\left(\lambda, \eta_{1}, \frac{\eta_{1} \eta_{2}}{\lambda}\right)
$$


with $\eta_{2} \leq \lambda \leq \eta_{1}$. For any $\lambda$ in the interval $\left[\eta_{2}, \eta_{1}\right]$, Theorem 2 shows that $\mathrm{SO}(3) \boldsymbol{W}_{1}(\lambda)$ and $\mathrm{SO}(3) \boldsymbol{W}_{2}(\lambda)$ are rank-1 connected. Using the solution of the two-well problem, it follows that $Q \mathcal{K}$ contains the tensor

$$
\left(\begin{array}{ccc}
u_{11} & u_{12} & 0 \\
u_{12} & u_{22} & 0 \\
0 & 0 & \frac{\eta_{1} \eta_{2}}{\lambda}
\end{array}\right)
$$

for any $\left(u_{11}, u_{22}, u_{12}\right)$ verifying

$$
u_{11} u_{22}-u_{12}^{2}=\eta_{1} \lambda, u_{11}^{2}+u_{22}^{2}+2 u_{12}^{2}+2\left|u_{12}\left(u_{11}+u_{22}\right)\right| \leq \eta_{1}^{2}+\lambda^{2} .
$$

For any given $u_{12}^{*}$, we denote by $\mathcal{U}^{-}\left(u_{12}^{*}\right)$ the set of tensors satisfying the relations (4.32)-(4.33) with $u_{12}=u_{12}^{*}$. The set $\mathcal{U}^{-}\left(u_{12}^{*}\right)$ is a lower bound on $\mathcal{U}\left(u_{12}^{*}\right)$. Note that $\mathcal{U}^{-}\left(u_{12}^{*}\right)$ is not empty as long as $\left|u_{12}\right| \leq u_{12}^{0}$ where $u_{12}^{0}$ is defined in (3.8). Theorem 3 shows that any given $\overline{\boldsymbol{U}}$ in $\mathcal{U}^{-}\left(u_{12}^{*}\right)$ is realized by a second-rank laminate involving deformation gradients in $\mathrm{SO}(3) \boldsymbol{W}_{1}(\lambda) \cup$ $\mathrm{SO}(3) \boldsymbol{W}_{2}(\lambda)$. Since deformation gradients in $\mathrm{SO}(3) \boldsymbol{W}_{i}(\lambda)$ are themselves realized by second-rank laminates (with deformation gradients in $\mathrm{SO}(3) \boldsymbol{U}_{2} \cup$ $\mathrm{SO}(3) \boldsymbol{U}_{3}$ and $\mathrm{SO}(3) \boldsymbol{U}_{1} \cup \mathrm{SO}(3) \boldsymbol{U}_{3}$ respectively), possible microstructures realizing any given $\overline{\boldsymbol{U}}$ in $\mathcal{U}^{-}\left(u_{12}^{*}\right)$ take the form of fourth-rank laminates, involving the three wells.

Applying the restrictions (4.25) to positive tensors of the form (4.29) yields an upper bound on $\mathcal{U}\left(u_{12}^{*}\right)$. That upper bound is denoted by $\mathcal{U}^{+}\left(u_{12}^{*}\right)$ and its boundary is represented on Fig. 2 for several values of $u_{12}^{*}$. It is found that $\mathcal{U}^{+}\left(u_{12}^{*}\right)$ collapses to the empty set when $u_{12}^{*}$ exceeds a certain threshold $u_{12}^{1}$. Some lengthy calculations based on expressions (4.25) shows that $u_{12}^{1}$ is actually equal to the value $u_{12}^{0}$ in (3.8).

There is generally a gap between the sets $\mathcal{U}^{-}\left(u_{12}^{*}\right)$ and $\mathcal{U}^{+}\left(u_{12}^{*}\right)$, as shown in Fig. 3 where $\mathcal{U}^{-}\left(u_{12}^{*}\right)$ is represented as a dotted area. Observe in Fig.3 that the boundaries of $\mathcal{U}^{-}\left(u_{12}^{*}\right)$ and $\mathcal{U}^{+}\left(u_{12}^{*}\right)$ have a non empty intersection. It can be verified that this intersection is formed by the deformation gradients in $Q\left(\mathrm{SO}(3) \boldsymbol{U}_{2} \cup \mathrm{SO}(3) \boldsymbol{U}_{3}\right)$. The dashed lines in Fig. 3 represent the bound obtained when replacing $\left(A_{k}^{\prime}(\overline{\boldsymbol{F}}), B_{k}^{\prime}(\overline{\boldsymbol{F}})\right)$ with $\left(A_{k}(\overline{\boldsymbol{F}}), B_{k}(\overline{\boldsymbol{F}})\right)$ in $(4.25)$, thus illustrating the improvement obtained by considering tensors $(\boldsymbol{\tau}, \boldsymbol{a})$ of the form (4.8) in the optimization.

The case $u_{12}^{*}=0$ is a special situation where the $\operatorname{sets} \mathcal{U}^{-}\left(u_{12}^{*}\right)$ and $\mathcal{U}^{+}\left(u_{12}^{*}\right)$ are equal, thus giving the exact value of $\mathcal{U}(0)$. The set of deformations $\mathcal{U}(0)$ corresponds to dilatations along the principal axis of the crystallographic 


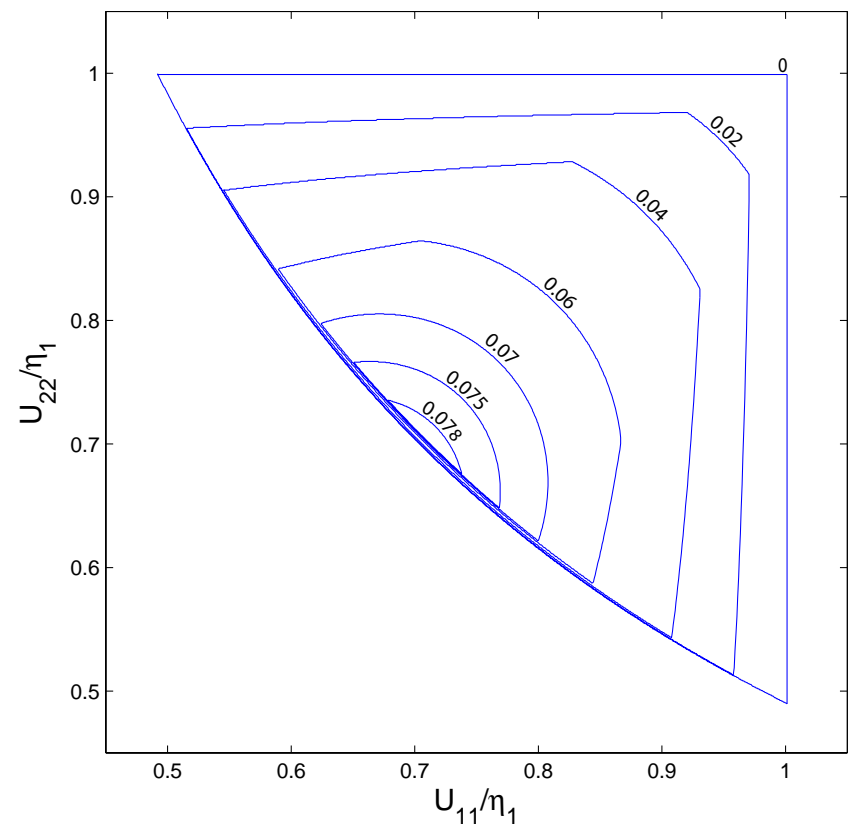

Figure 2: The set $\mathcal{U}^{+}\left(u_{12}^{*}\right)$ for several values of $u_{12}^{*}$. The ratio $\eta_{2} / \eta_{1}$ is set to 0.5 .

structure. It is represented in Fig. 2 in the case $\eta_{2} / \eta_{1}=0.5$, and in Fig. 4 (left) for $\mathrm{MnCu}\left(\eta_{2} / \eta_{1}=0.9561\right)$. When $u_{12}^{*}=0$, the restrictions (4.25) defining the bound $\mathcal{U}^{+}(0)$ reduce to

$$
\eta_{2} \leq u_{11} \leq \eta_{1}, \eta_{2} \leq u_{22} \leq \eta_{1}, \eta_{2} \eta_{1} \leq u_{11} u_{22}
$$

The lower bound $\mathcal{U}^{-}(0)$ obtained from Eqs. (4.32)-(4.33) is

$$
\mathcal{U}^{-}(0)=\left\{\operatorname{diag}\left(r, \eta_{1} \lambda / r, \eta_{1} \eta_{2} / \lambda\right), \eta_{2} \leq \lambda \leq \eta_{1}, \lambda \leq r \leq \eta_{1}\right\}
$$

It is easy to verify from these expressions that $\mathcal{U}^{+}(0)=\mathcal{U}^{-}(0)$.

For a given $\overline{\boldsymbol{U}}$ in $\mathcal{U}(0)$, there is in general neither unicity of the microstructure realizing $\overline{\boldsymbol{U}}$, nor of the volume fractions. Using the relation (4.23), the volume fractions $\boldsymbol{\theta}$ corresponding to a fixed $\overline{\boldsymbol{U}}=\operatorname{diag}\left(u_{i i}\right)$ in $\mathcal{U}(0)$ are found to verify

$$
\frac{\eta_{2}}{\eta_{1}^{2}-\eta_{2}^{2}}\left(\frac{\eta_{1}^{2}}{u_{i i}}-u_{i i}\right) \leq \theta_{i} \leq 1-\frac{\eta_{1}}{\eta_{1}^{2}-\eta_{2}^{2}}\left(u_{i i}-\frac{\eta_{2}^{2}}{u_{i i}}\right)
$$



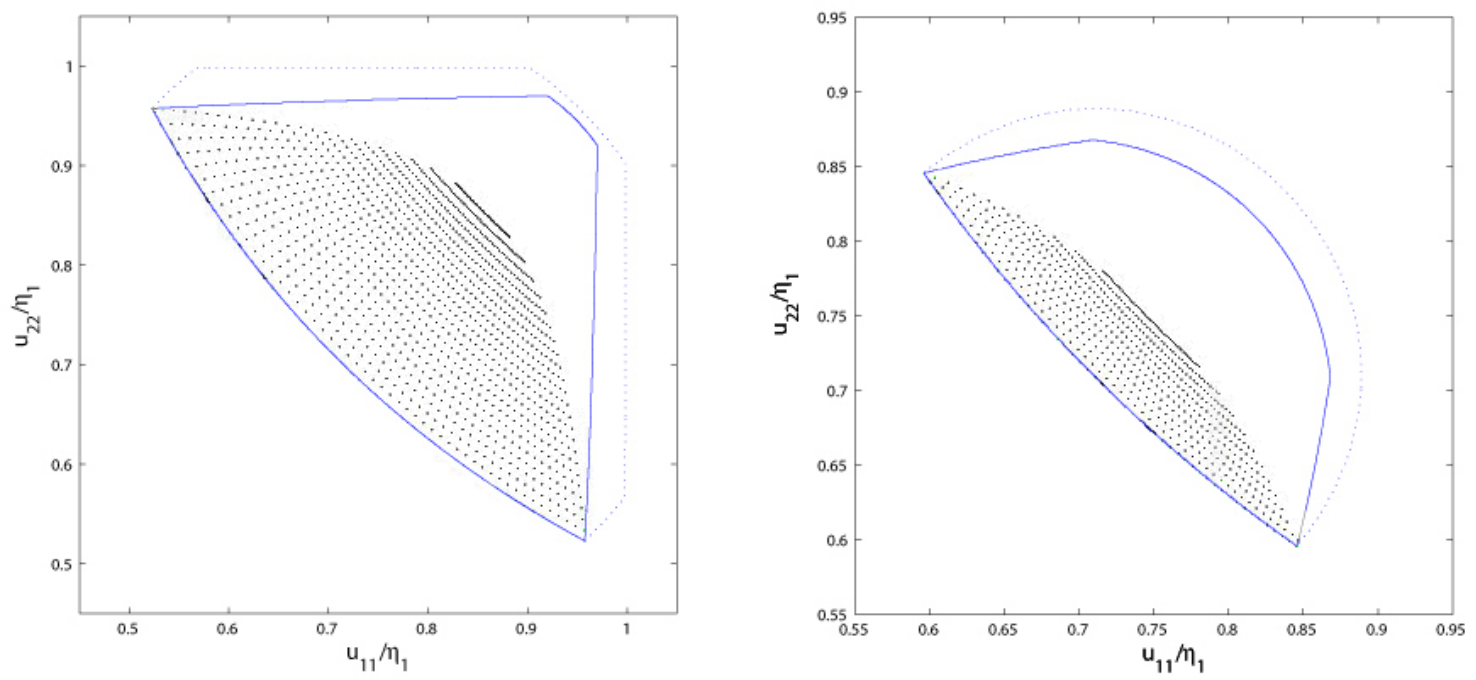

Figure 3: Representation of the set $\mathcal{U}\left(u_{12}^{*}\right)$ for the three-well problem, with $\eta_{2} / \eta_{1}=0.5$ and $u_{12}^{*} / \eta_{1}=0.02$ (left); $u_{12}^{*} / \eta_{1}=0.06$ (right).

which is complemented by the condition $\sum_{i} \theta_{i}=1$. For example, the volume fractions satisfying (4.35) are represented on Fig. 4 (right) for a particular deformation gradient in $\mathcal{U}(0)$ (point labeled A in Fig. 4 (left)).

As a further illustration of the restrictions obtained on $Q \mathcal{K}$, consider deformation gradients $\overline{\boldsymbol{F}}(\omega, \delta)$ of the form

$$
\overline{\boldsymbol{F}}(\omega, \delta)=\left(\eta_{1}^{2} \eta_{2}\right)^{1 / 3}\left(\boldsymbol{I}+\delta \boldsymbol{v}(\omega) \otimes \boldsymbol{v}^{\prime}(\omega)\right)
$$

where $\boldsymbol{v}(\omega)=\cos \omega \boldsymbol{v}_{1}+\sin \omega \boldsymbol{v}_{2}$ and $\boldsymbol{v}^{\prime}(\omega)=-\sin \omega \boldsymbol{v}_{1}+\cos \omega \boldsymbol{v}_{2}$. The deformation gradient $\overline{\boldsymbol{F}}(\omega, \delta)$ can be realized by cooling down the material (so as to produce a self-accomodated state with a deformation gradient $\left.\left(\eta_{1}^{2} \eta_{2}\right)^{1 / 3} \boldsymbol{I}\right)$ and subsequently applying a simple shear of amplitude $\delta$ between the directions $\boldsymbol{v}(\omega)$ and $\boldsymbol{v}^{\prime}(\omega)$. Note that $\operatorname{det} \overline{\boldsymbol{F}}(\omega, \delta)=\eta_{1}^{2} \eta_{2}$, which is a necessary condition for $\overline{\boldsymbol{F}}(\omega, \delta)$ to be in $Q \mathcal{K}$. The shaded area on Fig. 5 represents the values $(\delta, \omega)$ for which $\overline{\boldsymbol{F}}(\omega, \delta)$ meets the requirements (4.25). For every $\omega$ in $[0, \pi]$, the conditions (4.25) translate into a lower and an upper bound on the shear amplitude $\delta$. A remarkable feature of these curves is the existence of localized peaks at specific values of $\omega$. This property is confirmed by consideration of lower bounds on $Q \mathcal{K}$. The construction (4.32)-(4.33) can indeed be used to find values of $(\omega, \delta)$ such that $\overline{\boldsymbol{F}}(\omega, \delta) \in Q \mathcal{K}$. Consider a tensor $\boldsymbol{U}$ satisfying (4.32)-(4.33) with $\lambda=\left(\eta_{1} \eta_{2}^{2}\right)^{1 / 3}$. The spectral decomposition of 

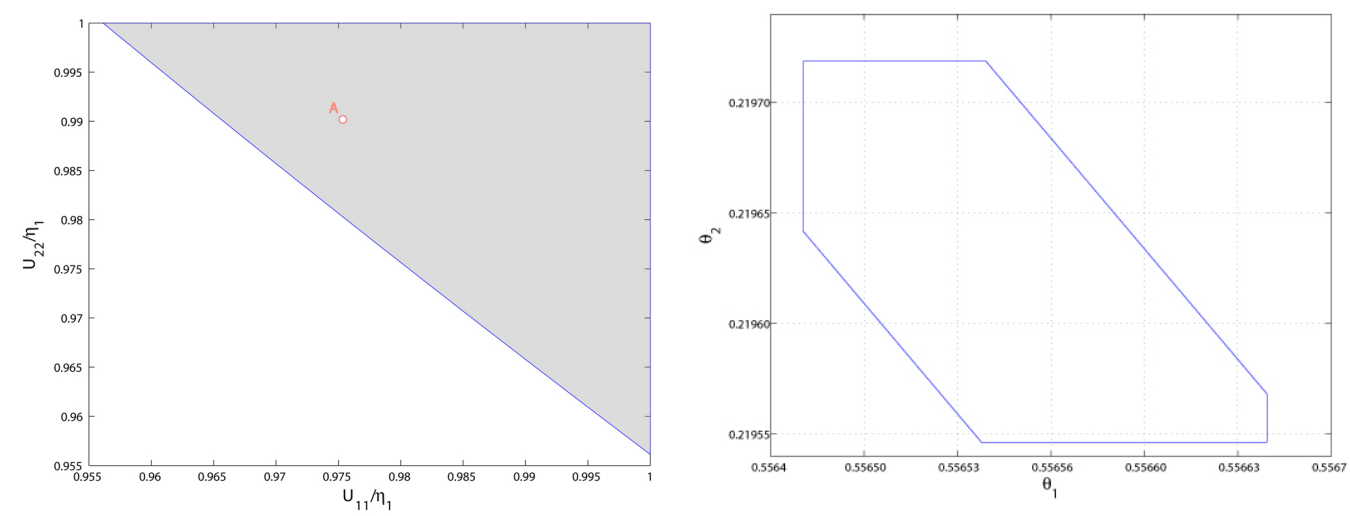

Figure 4: The set $\mathcal{U}(0)$ for $\mathrm{MnCu}$ (left); Bounds on the volume fractions for $\left(\frac{u_{11}}{\eta_{1}}, \frac{u_{22}}{\eta_{1}}\right)=$ $(0.975,0.99)$ (right).

$\boldsymbol{U}$ implies that there exists $\mu \geq 1$ and $\alpha \in[0,2 \pi]$ such that

$$
\boldsymbol{U}=\left(\eta_{1}^{2} \eta_{2}\right)^{1 / 3}\left(\mu \boldsymbol{w}_{1} \otimes \boldsymbol{w}_{1}+\frac{1}{\mu} \boldsymbol{w}_{2} \otimes \boldsymbol{w}_{2}+\boldsymbol{v}_{3} \otimes \boldsymbol{v}_{3}\right)
$$

with

$$
\boldsymbol{w}_{1}=\cos \alpha \boldsymbol{v}_{1}+\sin \alpha \boldsymbol{v}_{2}, \boldsymbol{w}_{2}=-\sin \alpha \boldsymbol{v}_{1}+\cos \alpha \boldsymbol{v}_{2} .
$$

Setting $\delta=\mu-\frac{1}{\mu}$ and $\beta=\arctan \mu$, it can be verified that $\overline{\boldsymbol{F}}(\pi-\beta+\alpha, \delta)=$ $\boldsymbol{R U}$ and $\overline{\boldsymbol{F}}(\beta-\pi+\alpha,-\delta)=\boldsymbol{R}^{\prime} \boldsymbol{U}$ for suitable rotations $\boldsymbol{R}$ and $\boldsymbol{R}^{\prime}$. This construction generates a lower bound on the set $\{(\omega, \delta) \mid \overline{\boldsymbol{F}}(\omega, \delta) \in Q \mathcal{K}\}$. That lower bound is represented by the dotted area in Fig. 5 and is found to have a similar shape as $P \mathcal{K}$.

Setting $\delta^{0}=\left(\eta_{1} / \eta_{2}\right)^{1 / 3}-\left(\eta_{1} / \eta_{2}\right)^{-1 / 3}$ and $\beta^{0}=\arctan \left(\left(\eta_{1} / \eta_{2}\right)^{1 / 3}\right)$, the peaks in Fig. 5 are found to correspond to

$$
(\omega, \delta)=\left(\pi-\beta^{0}, \delta^{0}\right),\left(\pi / 2-\beta^{0}, \delta^{0}\right),\left(\beta^{0}-\pi,-\delta^{0}\right),\left(\beta^{0}-\pi / 2,-\delta^{0}\right) .
$$

These values of $(\omega, \delta)$ are associated with the deformation gradients

$$
\left(\eta_{1}^{2} \eta_{2}\right)^{1 / 3} \operatorname{diag}\left(\left(\eta_{1} / \eta_{2}\right)^{1 / 3},\left(\eta_{1} / \eta_{2}\right)^{-1 / 3}, 1\right)
$$

and

$$
\left(\eta_{1}^{2} \eta_{2}\right)^{1 / 3} \operatorname{diag}\left(\left(\eta_{1} / \eta_{2}\right)^{-1 / 3},\left(\eta_{1} / \eta_{2}\right)^{1 / 3}, 1\right)
$$

Both these deformation gradients satisfy (4.32)-(4.33) and therefore are in $Q \mathcal{K}$. 
The dotted curves in Fig. 5 are the bounds given by the requirements (4.27), showing a significant difference between the bounds $P_{0} \mathcal{K}$ and $P \mathcal{K}$.

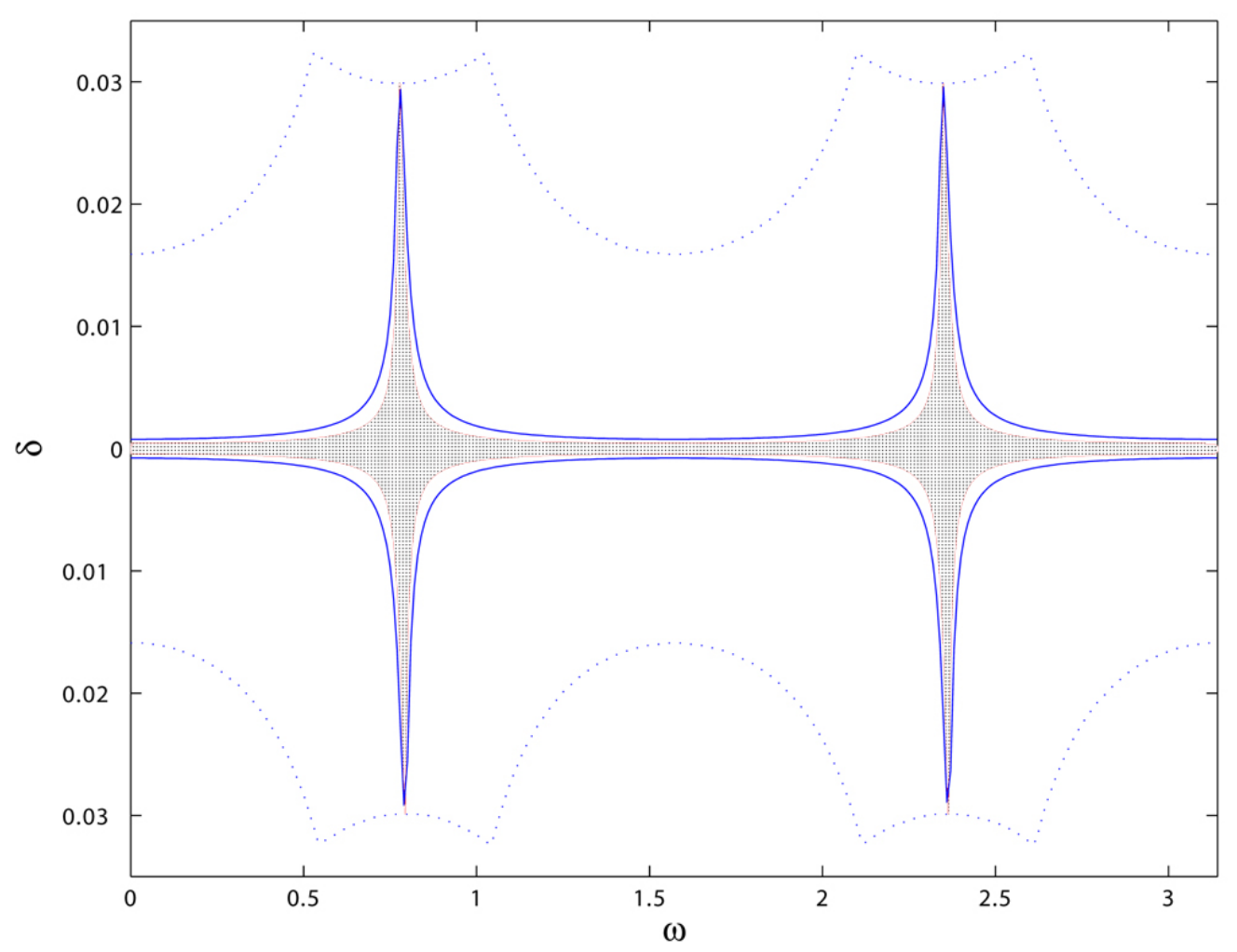

Figure 5: Bounds on the shear amplitude $\delta$ for $\mathrm{MnCu}$.

\subsection{Case $\eta_{2}<\mu_{1}<\eta_{1}$}

We now address the case where $\mu_{1} \neq \eta_{1}$ in (4.1). To keep the presentation short, we assume in the following that $\eta_{1}>\eta_{2}$, and we essentially focus our attention on the set $\mathcal{U}(0)$ of deformation gradients in $Q \mathcal{K}$ that are diagonal in the reference basis.

When $\eta_{2}<\mu_{1}<\eta_{1}$, it remains possible to exhibit microstructures involving the three wells, even though they are not necessarily pairwise rank-1 connected. Since $\mathrm{SO}(3) \boldsymbol{U}_{2}$ and $\mathrm{SO}(3) \boldsymbol{U}_{3}$ are rank-1 connected, $\mathcal{U}(0)$ indeed contains the tensors $\boldsymbol{W}_{1}(\lambda)$ defined in (4.31) for $\eta_{2} \leq \lambda \leq \eta_{1}$. Using Theorem 2, the tensor $\boldsymbol{W}_{1}\left(\mu_{1}\right)$ is found to have rank-1 connections with $\boldsymbol{U}_{1}$. 
Consequently, using the solution (3.6-3.7) of the two-well problem, $Q \mathcal{K}$ is seen to contain the tensors

$$
\operatorname{diag}\left(\lambda, \mu_{1}, \mu_{1} \mu_{2} / \lambda\right)
$$

for $\min \left(\mu_{2}, \eta_{1}\right) \leq \lambda \leq \max \left(\mu_{2}, \eta_{1}\right)$. Similarly, the tensor $\boldsymbol{W}_{1}\left(\eta_{1} \eta_{2} / \mu_{1}\right)$ is in $Q \mathcal{K}$ and has rank-1 connections with $\boldsymbol{U}_{1}$. Therefore, $Q \mathcal{K}$ also contains the tensors

$$
\operatorname{diag}\left(\lambda, \mu_{1} \mu_{2} / \lambda, \mu_{1}\right)
$$

for $\min \left(\mu_{2}, \eta_{1}\right) \leq \lambda \leq \max \left(\mu_{2}, \eta_{1}\right)$. For any fixed $\lambda$ between $\min \left(\mu_{2}, \eta_{1}\right)$ and $\max \left(\mu_{2}, \eta_{1}\right)$, use of Theorem 2 shows that the two tensors in (4.37) and (4.38) are rank-1 connected. Consequently $\mathcal{U}(0)$ contains the set of tensors

$$
\begin{aligned}
\left\{\operatorname{diag}\left(\lambda, \lambda^{\prime}, \mu_{1}^{2} \mu_{2} / \lambda \lambda^{\prime}\right) ; \quad\right. & \min \left(\mu_{2}, \eta_{1}\right) \leq \lambda \leq \max \left(\mu_{2}, \eta_{1}\right) \\
& \left.\min \left(\frac{\mu_{1} \mu_{2}}{\lambda}, \mu_{1}\right) \leq \lambda^{\prime} \leq \max \left(\frac{\mu_{1} \mu_{2}}{\lambda}, \mu_{1}\right)\right\}
\end{aligned}
$$

That set is depicted by the dotted area in Fig. 6. It has a 'triangular' shape, with corners $\boldsymbol{U}_{1}, \boldsymbol{W}_{1}\left(\mu_{1}\right)$ and $\boldsymbol{W}_{1}\left(\eta_{1} \eta_{2} / \mu_{1}\right)$. Invoking Theorem 3, any deformation gradient of the form (4.39) can be realized by a sixth-rank laminate involving the three wells.

We now explicit an upper bound on $Q \mathcal{K}$. For our purpose, it is sufficient to use (2.31) with $\left(\boldsymbol{v}, \boldsymbol{v}^{\prime}\right)=\left(\boldsymbol{v}_{i}, 0\right),\left(0, \boldsymbol{v}_{i}\right)$ and to optimize (2.25) with respect to tensors $(\boldsymbol{\tau}, \boldsymbol{a})$ of the form (4.8). Using (2.31) we obtain

$$
\begin{array}{ll}
\left\|\overline{\boldsymbol{F}} . \boldsymbol{v}_{1}\right\| \leq \max \left(\eta_{1}, \mu_{2}\right), & \left\|\overline{\boldsymbol{F}} \cdot \boldsymbol{v}_{2}\right\| \leq \eta_{1}, \quad\left\|\overline{\boldsymbol{F}} \cdot \boldsymbol{v}_{3}\right\| \leq \eta_{1}, \\
\left\|\overline{\boldsymbol{F}}^{*} \cdot \boldsymbol{v}_{1}\right\| \leq \max \left(\eta_{1} \eta_{2}, \mu_{1}^{2}\right), \quad\left\|\overline{\boldsymbol{F}}^{*} \cdot \boldsymbol{v}_{2}\right\| \leq \eta_{1}^{2}, \quad\left\|\overline{\boldsymbol{F}}^{*} \cdot \boldsymbol{v}_{3}\right\| \leq \eta_{1}^{2} .
\end{array}
$$

We now optimize (2.25) with respect to tensors $(\boldsymbol{\tau}, \boldsymbol{a})$ of the form $\boldsymbol{\tau}=$ $\sum_{i=1}^{3} \tau_{i} \boldsymbol{v}_{i} \otimes \boldsymbol{v}_{i}$ and $\boldsymbol{a}=\sum_{i=1}^{3} a_{i} \boldsymbol{v}_{i} \otimes \boldsymbol{v}_{i}$. The bound (2.25) gives

$$
0 \geq \sum_{i=1}^{3}\left(\bar{F}_{i i} \tau_{i}+\bar{F}_{i i}^{*} a_{i}\right)-\max \left(f\left(x_{1}, x_{2}, x_{3}\right), f\left(y_{1}, y_{2}, y_{3}\right), f\left(z_{1}, z_{2}, z_{3}\right)\right)
$$

with

$$
\begin{array}{lll}
x_{1}=\eta_{1} \tau_{1}+\eta_{1} \eta_{2} a_{1}, & x_{2}=\eta_{1} \tau_{2}+\eta_{1} \eta_{2} a_{2}, & x_{3}=\eta_{2} \tau_{3}+\eta_{1}^{2} a_{3}, \\
y_{1}=\mu_{2} \tau_{1}+\mu_{1}^{2} a_{1}, & y_{2}=\mu_{1} \tau_{2}+\mu_{1} \mu_{2} a_{2}, & y_{3}=\mu_{1} \tau_{3}+\mu_{1} \mu_{2} a_{3}, \\
z_{1}=x_{1}, & z_{2}=\eta_{2} \tau_{2}+\eta_{1}^{2} a_{2}, & z_{3}=\eta_{1} \tau_{3}+\eta_{1} \eta_{2} a_{3} .
\end{array}
$$


Using these relations to express $\left(\tau_{i}, a_{i}\right)$ as a function of $\left(z_{i}, y_{i}\right)$, the inequality (4.41) can be rewritten as

$$
0 \geq \sum_{i=1}^{3}\left(z_{i} p_{i}(\overline{\boldsymbol{F}})+y_{i} q_{i}(\overline{\boldsymbol{F}})\right)-\max \left(f\left(x_{1}, x_{2}, x_{3}\right), f\left(y_{1}, y_{2}, y_{3}\right), f\left(z_{1}, z_{2}, z_{3}\right)\right)
$$

where

$$
\begin{array}{ll}
p_{1}(\overline{\boldsymbol{F}})=\frac{\eta_{1}}{\eta_{1}^{2}-\mu_{2}^{2}}\left(\bar{F}_{11}-\frac{\mu_{2}}{\mu_{1}^{2}} \bar{F}_{11}^{*}\right), & q_{1}(\overline{\boldsymbol{F}})=\frac{-\mu_{2}}{\eta_{1}^{2}-\mu_{2}^{2}}\left(\bar{F}_{11}-\frac{1}{\eta_{2}} \bar{F}_{11}^{*}\right), \\
p_{2}(\overline{\boldsymbol{F}})=\frac{\eta_{2}}{\eta_{2}^{2}-\mu_{1}^{2}}\left(\bar{F}_{22}-\frac{1}{\mu_{2}} \bar{F}_{22}^{*}\right), & q_{2}(\overline{\boldsymbol{F}})=\frac{-\mu_{1}}{\eta_{2}^{2}-\mu_{1}^{2}}\left(\bar{F}_{22}-\frac{\eta_{2}}{\eta_{1}^{2}} \bar{F}_{22}^{*}\right), \\
p_{3}(\overline{\boldsymbol{F}})=\frac{\eta_{1}}{\eta_{1}^{2}-\mu_{1}^{2}}\left(\bar{F}_{33}-\frac{1}{\mu_{2}} \bar{F}_{33}^{*}\right), & q_{3}(\overline{\boldsymbol{F}})=\frac{-\mu_{1}}{\eta_{1}^{2}-\mu_{1}^{2}}\left(\bar{F}_{33}-\frac{1}{\eta_{2}} \bar{F}_{33}^{*}\right) .
\end{array}
$$

Note from (4.42) that the parameters $\left(x_{i}, y_{i}, z_{i}\right)$ are not independent. For $i \in\{2,3\}$ we can write

$$
x_{i}=\alpha_{i} z_{i}+\beta_{i} y_{i}
$$

with

$$
\alpha_{3}=\frac{\eta_{1}\left(\eta_{2}^{2}-\mu_{1}^{2}\right)}{\eta_{2}\left(\eta_{1}^{2}-\mu_{1}^{2}\right)}, \beta_{3}=\frac{\mu_{1}\left(\eta_{1}^{2}-\eta_{2}^{2}\right)}{\eta_{2}\left(\eta_{1}^{2}-\mu_{1}^{2}\right)}, \alpha_{2}=\frac{1}{\alpha_{3}}, \beta_{2}=-\frac{\beta_{3}}{\alpha_{3}} .
$$

The six parameters $\left(z_{i}, y_{i}\right)_{1 \leq i \leq 3}$ can be chosen arbitrarily. Maximizing the right-hand side of (4.43) with respect to $\left(z_{i}, y_{i}\right)_{1 \leq i \leq 3}$ shows that the optimal values are

$$
z_{1}=y_{1}=1, z_{2}=-z_{3}=\epsilon, y_{2}=-y_{3}=\epsilon \frac{\mu_{1}^{2}+\eta_{1} \eta_{2}}{\mu_{1}\left(\eta_{1}+\eta_{2}\right)}
$$

where $\epsilon= \pm 1$. Note in particular that those values are such that $f\left(x_{1}, x_{2}, x_{3}\right)=$ $f\left(y_{1}, y_{2}, y_{3}\right)=f\left(z_{1}, z_{2}, z_{3}\right)=1$. The expression (4.43) becomes

$$
1 \geq p_{1}(\overline{\boldsymbol{F}})+q_{1}(\overline{\boldsymbol{F}})+\epsilon\left(p_{2}(\overline{\boldsymbol{F}})-p_{3}(\overline{\boldsymbol{F}})\right)+\epsilon \frac{\mu_{1}^{2}+\eta_{1} \eta_{2}}{\mu_{1}\left(\eta_{1}+\eta_{2}\right)}\left(q_{2}(\overline{\boldsymbol{F}})-q_{3}(\overline{\boldsymbol{F}})\right) .
$$

This inequality corresponds to the use of diagonal tensors $(\boldsymbol{\tau}, \boldsymbol{a})$ in $(2.25)$. Optimizing with respect to all tensors of the form (4.8) shows that (4.48) has to be satisfied for all $\boldsymbol{R} . \overline{\boldsymbol{F}}$ where $\boldsymbol{R} \in \mathrm{SO}(3)$. It is possible to write the corresponding restrictions on $Q \mathcal{K}$ in terms of the function $\Phi$ introduced in 
(2.21), but for the sake of conciseness those developments are not reported here. In the case where $\overline{\boldsymbol{F}}=\operatorname{diag}\left(u_{i i}\right)$ with $u_{i i} \geq 0$ and $\operatorname{det} \overline{\boldsymbol{F}}=\eta_{1}^{2} \eta_{2}$, some algebraic manipulations allow the inequality (4.48) to be rewritten as

$$
0 \geq\left(u_{11}-\eta_{1}\right)\left(-\frac{\epsilon}{\eta_{1}\left(\eta_{1}+\eta_{2}\right)} u_{11} u_{22}^{2}+\frac{1}{\eta_{1}+\mu_{2}}\left(u_{11}-\mu_{2}\right) u_{22}+\epsilon \frac{\eta_{1} \eta_{2}}{\eta_{1}+\eta_{2}}\right) .
$$

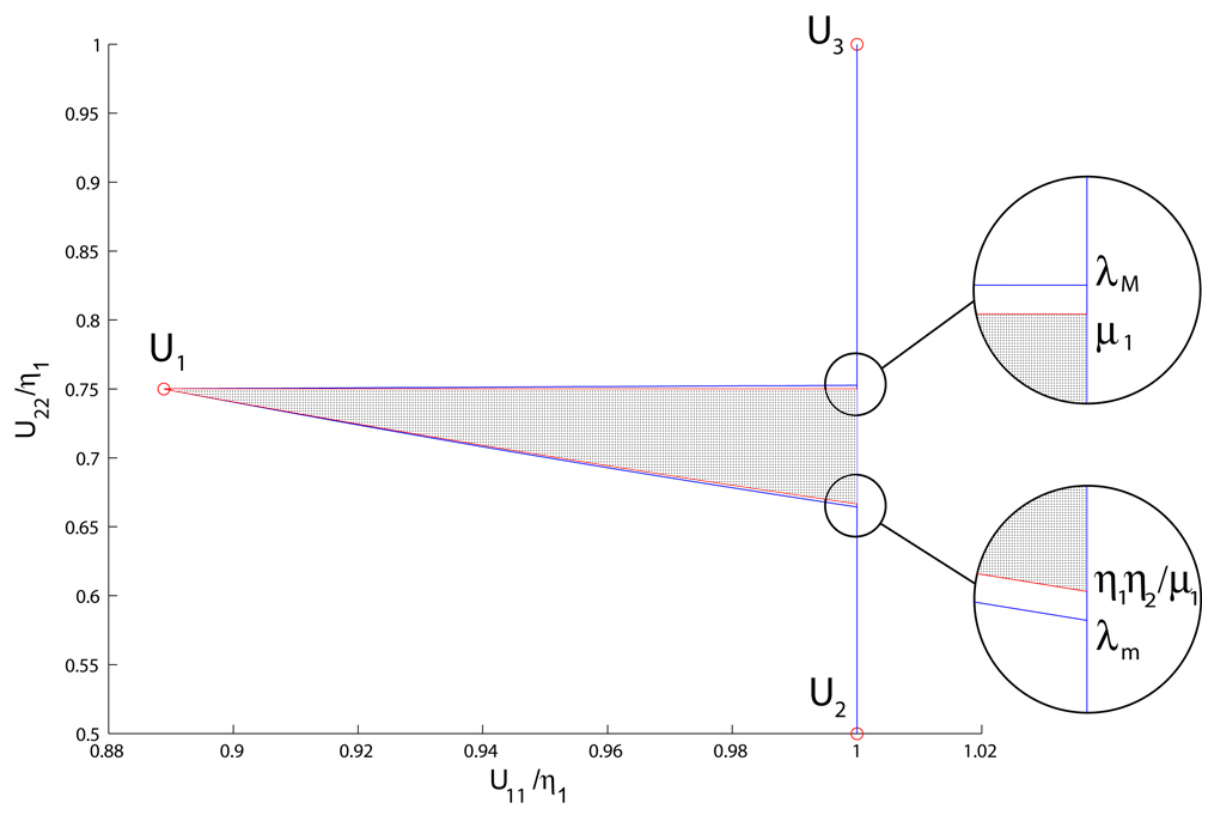

Figure 6: Lower and upper bounds on $\mathcal{U}(0)$ in the case $\eta_{2} / \eta_{1}=0.5, \mu_{1} / \eta_{1}=0.75$.

We denote by $\mathcal{U}^{+}(0)$ the set of tensors $\operatorname{diag}\left(u_{i i}\right)$ satisfying (4.40) and (4.49). The set $\mathcal{U}^{+}(0)$ is an upper bound on $\mathcal{U}(0)$. The set obtained is the union of the segment $\left[\boldsymbol{U}_{1}, \boldsymbol{U}_{3}\right]$ with a 'triangular' area, as depicted in Fig. 6 in the case $\eta_{2} / \eta_{1}=0.5, \mu_{1} / \eta_{1}=0.75$. The corners of that triangular area are $\boldsymbol{U}_{1}, \boldsymbol{W}_{1}\left(\lambda_{m}\right), \boldsymbol{W}_{1}\left(\lambda_{M}\right)$ where

$$
\lambda_{m}=\frac{\eta_{1}+\eta_{2}}{2}\left(-\frac{\left|\eta_{1}-\mu_{2}\right|}{\eta_{1}+\mu_{2}}+\sqrt{\Delta}\right), \lambda_{M}=\frac{\eta_{1}+\eta_{2}}{2}\left(\frac{\left|\eta_{1}-\mu_{2}\right|}{\eta_{1}+\mu_{2}}+\sqrt{\Delta}\right)
$$

and $\Delta=\left(\eta_{1}-\mu_{2}\right)^{2} /\left(\eta_{1}+\mu_{2}\right)^{2}+4 \eta_{1} \eta_{2} /\left(\eta_{1}+\eta_{2}\right)^{2}$. The upper bound $\mathcal{U}^{+}(0)$ is found to be very close to the lower bound defined by (4.39). A measure of 
the gap between those two sets is obtained by comparing $\left|\mu_{1}-\eta_{1} \eta_{2} / \mu_{1}\right|$ with $\lambda_{M}-\lambda_{m}$ (see Fig.6). We have

$$
\frac{\lambda_{M}-\lambda_{m}}{\left|\mu_{1}-\eta_{1} \eta_{2} / \mu_{1}\right|}-1=\frac{\mu_{1}\left(\eta_{1}+\eta_{2}\right)}{\mu_{1}^{2}+\eta_{1} \eta_{2}}-1 .
$$

In the case depicted in Fig. 6, this scalar is approximatively equal to $5.88 \%$. Fig. 7 represents the upper bound $\mathcal{U}^{+}(0)$ for different values of $\mu_{1}$ in the interval $\left[\eta_{2}, \eta_{1}\right]$. Fig. 8 shows the evolution of $\lambda_{M}-\lambda_{m}$ and $\left|\mu_{1}-\eta_{1} \eta_{2} / \mu_{1}\right|$ as a function of $\mu_{1}$. Notice that the upper bound $\mathcal{U}^{+}(0)$ and the lower bound (4.39) coincide for $\mu_{1} \in\left\{\eta_{2}, \eta_{1}, \sqrt{\eta_{2} \eta_{1}}\right\}$. Those values of $\mu_{1}$ correspond to cases where the three wells are pairwise rank-1 connected.

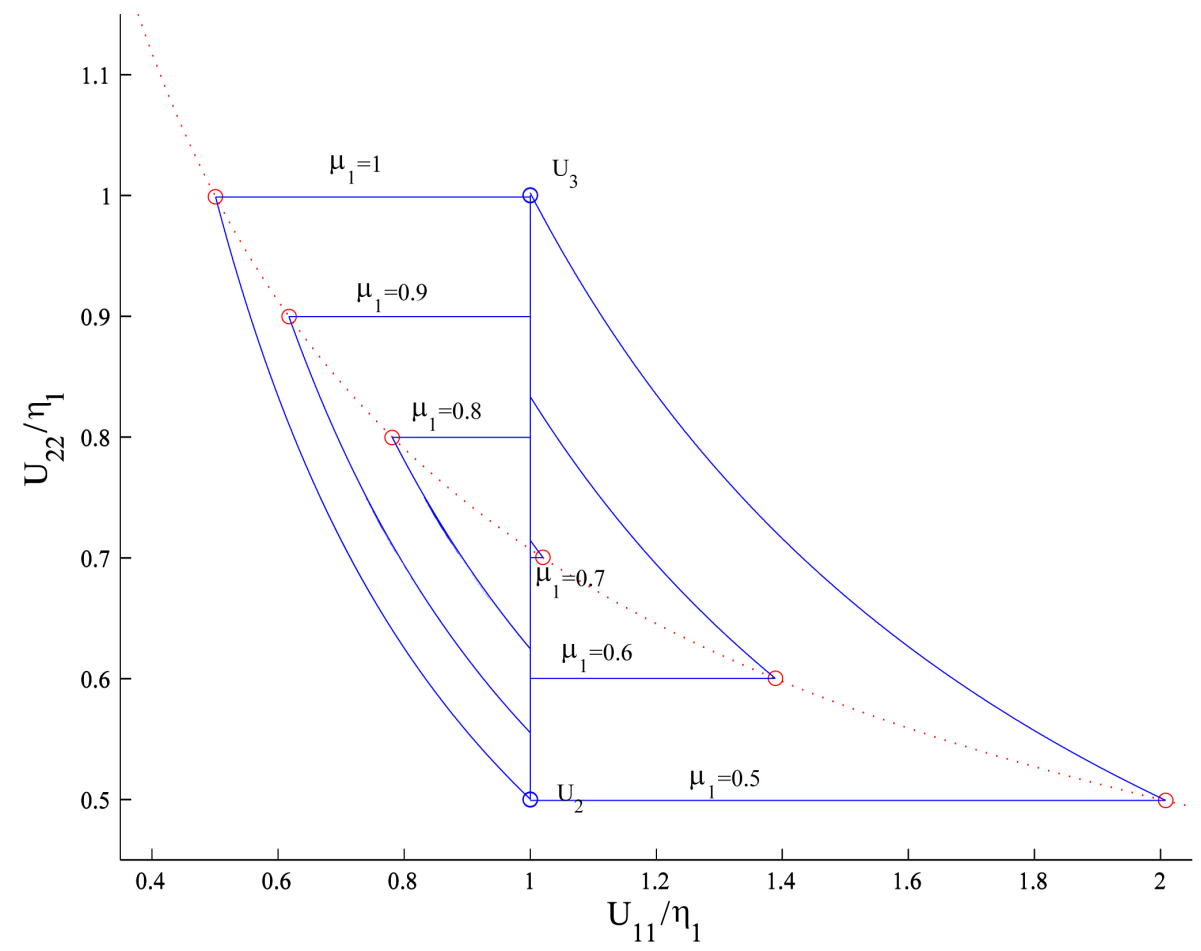

Figure 7: Evolution of the upper bound on $\mathcal{U}(0)$ as $\mu_{1}$ varies from $\eta_{2}=0.5$ to $\eta_{1}=1$.

\subsection{Case $\mu_{1} \notin\left[\eta_{2}, \eta_{1}\right]$}

When $\mu_{1} \notin\left[\eta_{2}, \eta_{1}\right]$, the considerations used in the previous section for constructing tensors in $\mathcal{U}(0)$ fail. The only tensors in $\mathcal{U}(0)$ which can be 


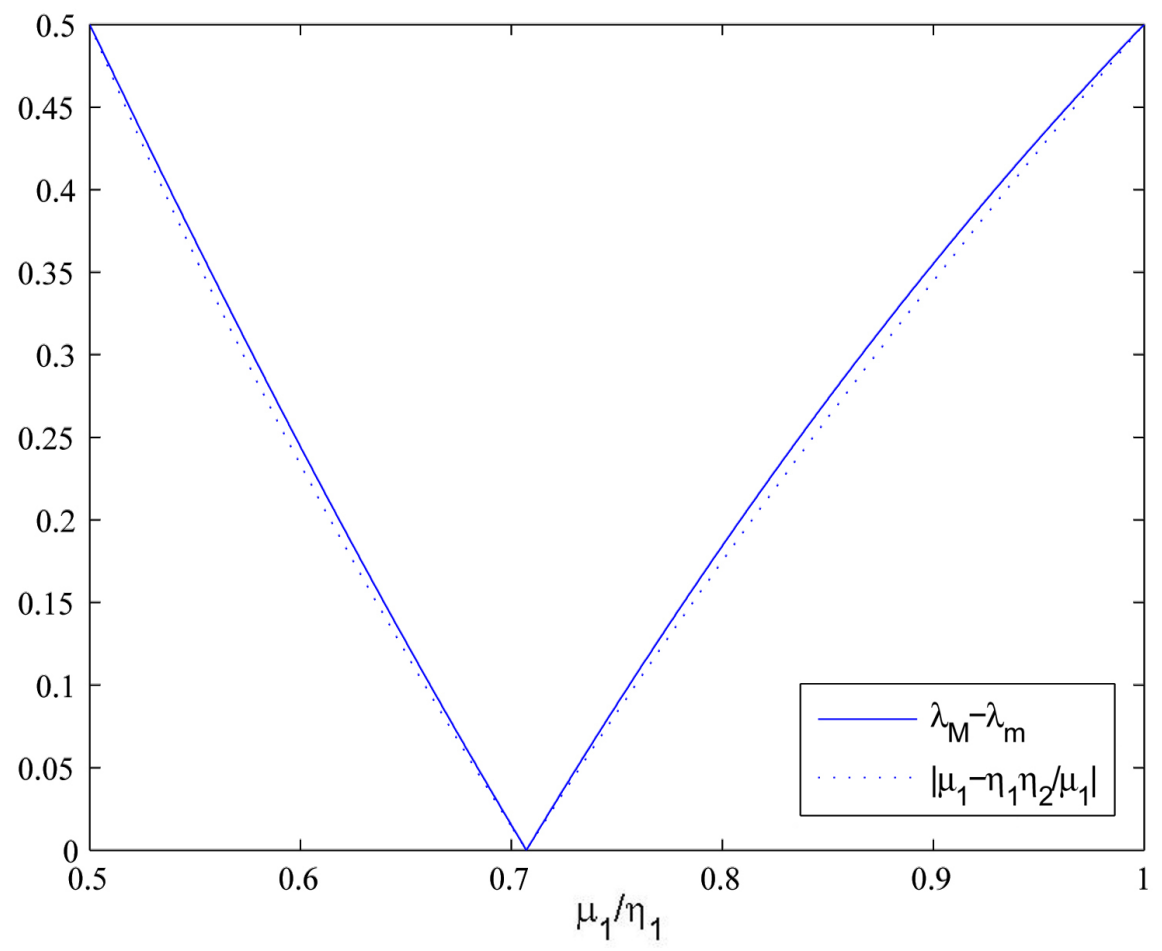

Figure 8: Comparison of the lower and upper bound on $\mathcal{U}(0)$.

simply exhibited are $\boldsymbol{U}_{1}$ and $\boldsymbol{W}_{1}(\lambda)$ for $\eta_{2} \leq \lambda \leq \eta_{1}$. This does not imply however that microstructures involving the three wells do not exist.

We assume in the following that $\mu_{1}>\eta_{1}>\eta_{2}$. Using (2.30) we obtain

$$
\begin{aligned}
& \left\|\overline{\boldsymbol{F}} \cdot \boldsymbol{v}_{1}\right\| \leq \eta_{1},\left\|\overline{\boldsymbol{F}}^{*} \cdot \boldsymbol{v}_{1}\right\| \leq \mu_{1}^{2}, \\
& \left\|\overline{\boldsymbol{F}} \cdot \boldsymbol{v}_{i}\right\| \leq \mu_{1},\left\|\overline{\boldsymbol{F}}^{*} \cdot \boldsymbol{v}_{i}\right\| \leq \eta_{1}^{2} \text { for } i=2,3 .
\end{aligned}
$$

Like in the previous subsection, additional information is obtained by optimizing (2.25) with respect to tensors $(\boldsymbol{\tau}, \boldsymbol{a})$ of the form (4.8). Carrying out this calculation shows that, compared to (4.50), the only additional information is obtained by using (4.43) with

$$
-z_{1}=z_{3}=\frac{\eta_{2} \mu_{1}+\eta_{1}^{2}}{\eta_{1}\left(\eta_{2}+\mu_{1}\right)}, z_{2}=-y_{1}=y_{2}=y_{3}=1 \text {. }
$$

Observe that $f\left(y_{1}, y_{2}, y_{3}\right)=f\left(z_{1}, z_{2}, z_{3}\right)$ for such values of $\left(y_{i}, z_{i}\right)$. Using (4.45)-(4.46), it can also be verified that $f\left(x_{1}, x_{2}, x_{3}\right)=1$. Substituting 
(4.51) in (4.43) yields

$1 \geq \frac{\eta_{2} \mu_{1}+\eta_{1}^{2}}{\eta_{1}\left(\eta_{2}+\mu_{1}\right)}\left(-p_{1}(\boldsymbol{R} . \overline{\boldsymbol{F}})+p_{3}(\boldsymbol{R} . \overline{\boldsymbol{F}})\right)+p_{2}(\boldsymbol{R} . \overline{\boldsymbol{F}})+q_{2}(\boldsymbol{R} . \overline{\boldsymbol{F}})+q_{3}(\boldsymbol{R} . \overline{\boldsymbol{F}})-q_{1}(\boldsymbol{R} . \overline{\boldsymbol{F}})$

for all $\boldsymbol{R} \in \mathrm{SO}(3)$.

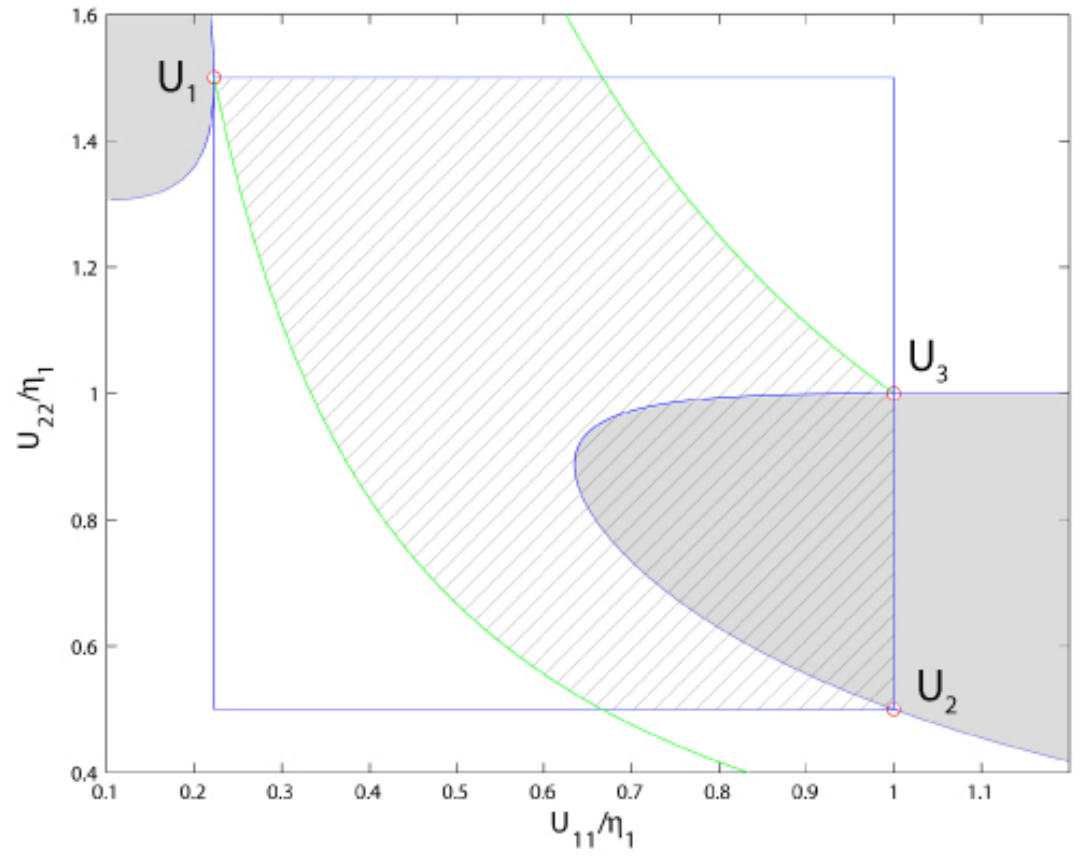

Figure 9: Upper bound on $\mathcal{U}(0)$ in the case $\eta_{1}<\mu_{1}$.

The tensors $\overline{\boldsymbol{F}}=\operatorname{diag}\left(u_{i i}\right)$ satisfying that inequality with $\boldsymbol{R}=\boldsymbol{I}$ are represented by the shaded gray area in Fig. 9 , in the case $\eta_{1}=1, \eta_{2}=0.5$, $\mu_{1}=1.5$. The intersection with the tensors satisfying (4.50) (represented by the hatched area in Fig. 9) defines an upper bound on $\mathcal{U}(0)$. In contrast with the case $\mu_{1} \leq \eta_{1}$, the set $\mathcal{U}(0)$ of diagonal strains in $Q \mathcal{K}$ is not a connected set $\left(\boldsymbol{U}_{1}\right.$ is an isolated point). Note that the upper bound obtained allows for mixture of the three phases. It is not clear whether such microstructures do really exist, or whether that result is due to a non optimality of the upper bound considered. Similar results can be obtained when $\mu_{1} \leq \eta_{2}$.

We close this study of the three-well problem by summarizing the main results that have been obtained. As long as $\mu_{1} \in\left[\eta_{2}, \eta_{1}\right]$, the set $\mathcal{U}(0)$ is 
connected (in the topological sense) and we can exhibit some laminate microstructures involving the three wells. The upper bound $\mathcal{U}^{+}(0)$ is found to be quite close to the lower bound (4.39) constructed from laminate microstructures. Those bounds are actually equal when the three wells are pairwise rank-1 connected, which happens not only for $\mu_{1}=\eta_{1}$ (cubic to tetragonal transformation) but also for $\mu_{1}=\eta_{2}$ and $\mu_{1}=\sqrt{\eta_{1} \eta_{2}}$. When $\mu_{1} \notin\left[\eta_{2}, \eta_{1}\right]$, it is no longer possible to simply exhibit microstructures involving the three wells. This coincides with a drastic change in the structure of $\mathcal{U}(0)$ : the deformation gradient $\boldsymbol{U}_{1}$ becomes an isolated point in $\mathcal{U}(0)$.

Although much attention has been devoted to tensors in $Q \mathcal{K}$ that are diagonal (in the reference basis), we note that the expressions (4.25) and (4.48) give an upper bound on the whole set $Q \mathcal{K}$, not just on its trace on the space of diagonal tensors.

\section{Reduction to two wells in cubic to monoclinic transformations}

In this section, we consider some implications of the bounds (2.30) for the twelve-well problems corresponding to cubic to monoclinic transformations. More precisely, we aim at deriving sufficient conditions on $\overline{\boldsymbol{F}}$ for the corresponding gradient Young measures to be supported on only two of the 12 wells. For the cubic to tetragonal transformation, Ball and James (1992)

showed that such a reduction to two wells is ensured if $\overline{\boldsymbol{F}}$ is a solution of a two-well problem involving two of the three variants.

Bhattacharya et al. (1999) studied the cubic to orthorombic-I transformation, in which case there are six wells, all pairwise rank-1 connected. These authors showed that, except for some very special values of the lattice parameters, the Young measure is unique (and supported on two wells) for any deformation gradient $\overline{\boldsymbol{F}}$ that can be realized by a first-rank laminate.

In this section, we investigate the extent to which such results can be generalized to the cubic to monoclinic transformations. There are two types of cubic to monoclinic transformations (classically referred to as cubic to monoclinic-I and cubic to monoclinic-II). The methodology employed can actually be applied to any $n$-well problem $\mathcal{K}=\cup_{r=1}^{n} \mathrm{SO}(3) \boldsymbol{U}_{r}$ where the transformation strains $\boldsymbol{U}_{r}$ are symmetric positive with the same determinant. Consider a given deformation gradient $\overline{\boldsymbol{F}}$ and two rank-1 connected transformation strains $\left(\boldsymbol{U}_{i}, \boldsymbol{U}_{j}\right)$. Assume that one can find a vector $\boldsymbol{v}$ verifying

$$
\left(\boldsymbol{U}_{i}^{2}-\boldsymbol{U}_{j}^{2}\right) . \boldsymbol{v}=0,\left\|\boldsymbol{U}_{k} \cdot \boldsymbol{v}\right\|<\left\|\boldsymbol{U}_{i} \cdot \boldsymbol{v}\right\| \text { for all } k \neq i, j .
$$


Noting that $\left\|\boldsymbol{U}_{i} . \boldsymbol{v}\right\|=\left\|\boldsymbol{U}_{j} . \boldsymbol{v}\right\|$ and applying the inequality (2.30) with $\boldsymbol{v}^{\prime}=0$, we obtain

$$
\begin{aligned}
\|\overline{\boldsymbol{F}} . \boldsymbol{v}\| & \leq\left(\theta_{i}+\theta_{j}\right)\left\|\boldsymbol{U}_{i} \cdot \boldsymbol{v}\right\|+\sum_{k \neq i, j} \theta_{k}\left\|\boldsymbol{U}_{k} \cdot \boldsymbol{v}\right\| \\
& \leq\left(\theta_{i}+\theta_{j}\right)\left(\left\|\boldsymbol{U}_{i} \cdot \boldsymbol{v}\right\|-\sup _{k \neq i, j}\left\|\boldsymbol{U}_{k} \cdot \boldsymbol{v}\right\|\right)+\sup _{k \neq i, j}\left\|\boldsymbol{U}_{k} \cdot \boldsymbol{v}\right\|,
\end{aligned}
$$

which can be rewritten as

$$
\frac{\|\overline{\boldsymbol{F}} \cdot \boldsymbol{v}\|-\sup _{k \neq i, j}\left\|\boldsymbol{U}_{k} \cdot \boldsymbol{v}\right\|}{\left\|\boldsymbol{U}_{i} \cdot \boldsymbol{v}\right\|-\sup _{k \neq i, j}\left\|\boldsymbol{U}_{j} \cdot \boldsymbol{v}\right\|} \leq \theta_{i}+\theta_{j}
$$

Therefore, if $\overline{\boldsymbol{F}}$ is such that $\|\overline{\boldsymbol{F}} \cdot \boldsymbol{v}\|=\left\|\boldsymbol{U}{ }_{i} \cdot \boldsymbol{v}\right\|$ then $\theta_{i}+\theta_{j}=1$ and $\theta_{k}=0$ for $k \neq i, j$. Possible microstructures realizing $\overline{\boldsymbol{F}}$ are thus necessarily restricted to the wells $\mathrm{SO}(3) \boldsymbol{U}_{i}$ and $\mathrm{SO}(3) \boldsymbol{U}_{j}$. Note from Theorem 4 that the condition $\|\overline{\boldsymbol{F}} \boldsymbol{v} \boldsymbol{v}\|=\left\|\boldsymbol{U}_{i} \cdot \boldsymbol{v}\right\|$ is satisfied for all $\overline{\boldsymbol{F}}$ in $Q\left(\mathrm{SO}(3) \boldsymbol{U}_{i} \cup \mathrm{SO}(3) \boldsymbol{U}_{j}\right)$, i.e. for any $\overline{\boldsymbol{F}}$ solution of the two-well problem involving variants $i$ and $j$.

In the same fashion, assume that one can find a vector $\boldsymbol{w}$ verifying

$$
\left(\boldsymbol{U}_{i}^{*, 2}-\boldsymbol{U}_{j}^{*, 2}\right) \cdot \boldsymbol{w}=0,\left\|\boldsymbol{U}_{k}^{*} \cdot \boldsymbol{w}\right\|<\left\|\boldsymbol{U}_{i}^{*} \cdot \boldsymbol{w}\right\| \text { for all } k \neq i, j
$$

A similar reasoning shows that if $\left\|\overline{\boldsymbol{F}}^{*} \cdot \boldsymbol{w}\right\|=\left\|\boldsymbol{U}_{i}^{*} \cdot \boldsymbol{w}\right\|$-which by Theorem 4 is satisfied for all $\overline{\boldsymbol{F}}$ in $Q\left(\mathrm{SO}(3) \boldsymbol{U}_{i} \cup \mathrm{SO}(3) \boldsymbol{U}_{j}\right)$ - then the gradient Young measure is supported on $\mathrm{SO}(3) \boldsymbol{U}_{i} \cup \mathrm{SO}(3) \boldsymbol{U}_{j}$.

Since we know from Theorem 4 that $\operatorname{Ker}\left(\boldsymbol{U}_{i}^{2}-\boldsymbol{U}_{j}^{2}\right)$ and $\operatorname{Ker}\left(\boldsymbol{U}_{i}^{*, 2}-\boldsymbol{U}_{j}^{*, 2}\right)$ are of dimension 1, it is easy to check if (5.1) or (5.4) can be satisfied. Even if neither (5.1) nor (5.4) holds, restriction to two wells may still be ensured for special deformation gradients in $Q\left(\mathrm{SO}(3) \boldsymbol{U}_{i} \cup \mathrm{SO}(3) \boldsymbol{U}_{j}\right)$. Consider in particular deformation gradients that are realized by first-rank laminates. Such deformation gradients $\overline{\boldsymbol{F}}$ are of the form

$$
\overline{\boldsymbol{F}}=\boldsymbol{U}_{i}+\lambda \boldsymbol{a} \otimes \boldsymbol{n}
$$

where $0 \leq \lambda \leq 1$ and $(\boldsymbol{a}, \boldsymbol{n})$ is solution of the twinning equation $\boldsymbol{R} \boldsymbol{U}_{j}-\boldsymbol{U}_{i}=$ $\boldsymbol{a} \otimes \boldsymbol{n}$. Assume one can find a vector $\boldsymbol{v}$ such that

$$
\boldsymbol{v} . \boldsymbol{n}=0,\left\|\boldsymbol{U}_{k} . \boldsymbol{v}\right\|<\left\|\boldsymbol{U}_{i} \cdot \boldsymbol{v}\right\| \text { for all } k \neq i, j .
$$

Since $\boldsymbol{v}$ is orthogonal to $\boldsymbol{n}$, we have $\|\overline{\boldsymbol{F}} \cdot \boldsymbol{v}\|=\left\|\boldsymbol{U}_{i} \cdot \boldsymbol{v}\right\|=\left\|\boldsymbol{U}_{j} . \boldsymbol{v}\right\|$. Using the inequality (2.30) with such a vector $\boldsymbol{v}$ and with $\boldsymbol{v}^{\prime}=0$, we obtain that 
$\theta_{i}+\theta_{j}=1$. The reduction to two wells is thus recovered. Moreover, invoking Theorem 3, there is uniqueness of the gradient Young measure.

Since the equation $\boldsymbol{R} \boldsymbol{U}_{j}-\boldsymbol{U}_{i}=\boldsymbol{a} \otimes \boldsymbol{n}$ can be rewritten as $\boldsymbol{R} \boldsymbol{U}_{j}^{*}-\boldsymbol{U}_{i}^{*}=$ $-\operatorname{det} \boldsymbol{U}_{i}\left(\boldsymbol{R} \boldsymbol{U}_{j}\right)^{-T} \boldsymbol{n} \otimes \boldsymbol{U}_{i}^{-1} \boldsymbol{a}$ (provided $\operatorname{det} \boldsymbol{U}_{i}=\operatorname{det} \boldsymbol{U}_{j}$ ), similar conclusions are obtained if there exists a vector $\boldsymbol{w}$ verifying

$$
\boldsymbol{w} \cdot \boldsymbol{U}_{i}^{-1} \cdot \boldsymbol{a}=0,\left\|\boldsymbol{U}_{k}^{*} \cdot \boldsymbol{w}\right\|<\left\|\boldsymbol{U}_{i}^{*} \cdot \boldsymbol{w}\right\| \text { for all } k \neq i, j
$$

Conditions of the form (5.6) and (5.7) are essentially the arguments used by Bhattacharya et al. (1999) for proving the restriction to two wells in the cubic to orthorombic transformation. Let $\left(\boldsymbol{u}_{1}, \boldsymbol{u}_{2}\right)$ be an orthonormal basis

of the plane $\boldsymbol{n}^{\perp}$. Setting $\boldsymbol{v}(\omega)=\cos \omega \boldsymbol{u}_{1}+\sin \omega \boldsymbol{u}_{2}$, the condition (5.6) can be rewritten as

$$
\sup _{0 \leq \omega \leq \pi}\left\{\left\|\boldsymbol{U}_{i} \cdot \boldsymbol{v}(\omega)\right\|^{2}-\sup _{k \neq i, j}\left\|\boldsymbol{U}_{k} \cdot \boldsymbol{v}(\omega)\right\|^{2}\right\}>0 .
$$

In a similar fashion, considering an orthonormal basis $\left(\boldsymbol{w}_{1}, \boldsymbol{w}_{2}\right)$ of the plane $\left(\boldsymbol{U}_{i}^{-1} \cdot \boldsymbol{a}\right)^{\perp}$ and setting $\boldsymbol{w}(\omega)=\cos \omega \boldsymbol{w}_{1}+\sin \omega \boldsymbol{w}_{2}$, the condition (5.7) is equivalent to

$$
\sup _{0 \leq \omega \leq \pi}\left\{\left\|\boldsymbol{U}_{i}^{*} \cdot \boldsymbol{v}(\omega)\right\|^{2}-\sup _{k \neq i, j}\left\|\boldsymbol{U}_{k}^{*} \cdot \boldsymbol{v}(\omega)\right\|^{2}\right\}>0
$$

For given values of $\left(\boldsymbol{U}_{1}, \cdots, \boldsymbol{U}_{n}\right)$, it can easily be checked numerically if (5.8) or (5.9) is satisfied. In the rest of this Section, the general procedure described so far is applied to the cubic to monoclinic transformations.

\subsection{Cubic to monoclinic-I transformation}

The twelve transformations strains in the cubic to monoclinic-I transformation are listed in Table 1 . The matrix representations in Table 1 are relative to the orthonormal basis $\left(\boldsymbol{v}_{1}, \boldsymbol{v}_{2}, \boldsymbol{v}_{3}\right)$ of the austenitic cubic lattice. In the following, we take $\alpha=1.0243, \beta=0.9563, \delta=0.058, \epsilon=-0.0427$. These values correspond to Ni-49.75Ti (Knowles and Smith, 1981). Using Theorem 2, each variant is found to have rank-1 connections with seven of the others. For instance, variant 1 has rank- 1 connections with variant $i$ for $i \in\{2,3,4,5,8,9,11\}$. Considering such a variant $i$, let us examine sufficient conditions on $\overline{\boldsymbol{F}}$ for the microstructure to be necessarily restricted to the wells $\mathrm{SO}(3) \boldsymbol{U}_{1}$ and $\mathrm{SO}(3) \boldsymbol{U}_{i}$. 


\begin{tabular}{|c|c|c|c|c|c|c|c|c|c|c|c|}
\hline \multicolumn{3}{|c|}{$\boldsymbol{U}_{1}$} & \multicolumn{3}{|c|}{$\boldsymbol{U}_{2}$} & \multicolumn{3}{|c|}{$\boldsymbol{U}_{3}$} & \multicolumn{3}{|c|}{$\boldsymbol{U}_{4}$} \\
\hline & $\epsilon$ & $\epsilon$ & $\beta$ & $-\epsilon$ & $-\epsilon$ & $\beta$ & $-\epsilon$ & $\epsilon$ & $\beta$ & $\epsilon$ & $-\epsilon$ \\
\hline & a & $\delta$ & $-\epsilon$ & $\alpha$ & $\delta$ & $-\epsilon$ & $\alpha$ & $-\delta$ & $\epsilon$ & $\alpha$ & $-\delta$ \\
\hline & $\delta$ & $\alpha$ & $-\epsilon$ & $\delta$ & $\alpha$ & $\epsilon$ & $-\delta$ & $\alpha$ & $-\epsilon$ & $-\delta$ & $\alpha$ \\
\hline \multicolumn{3}{|c|}{$\boldsymbol{U}_{5}$} & \multicolumn{3}{|c|}{$\boldsymbol{U}_{6}$} & \multicolumn{3}{|c|}{$\boldsymbol{U}_{7}$} & \multicolumn{3}{|c|}{$U_{8}$} \\
\hline & & $\delta$ & $\alpha$ & $-\epsilon$ & $\delta$ & $\alpha$ & $-\epsilon$ & $-\delta$ & $\alpha$ & $\epsilon$ & $-\delta$ \\
\hline & $\beta$ & $\epsilon$ & $-\epsilon$ & $\beta$ & $-\epsilon$ & $-\epsilon$ & $\beta$ & $\epsilon$ & $\epsilon$ & $\beta$ & $-\epsilon$ \\
\hline & $\epsilon$ & $\alpha$ & $\delta$ & $-\epsilon$ & $\alpha$ & $-\delta$ & $\epsilon$ & $\alpha$ & $-\delta$ & $-\epsilon$ & $\alpha$ \\
\hline \multicolumn{3}{|c|}{$\boldsymbol{U}_{9}$} & \multicolumn{3}{|c|}{$U_{10}$} & \multicolumn{3}{|c|}{$U_{11}$} & \multicolumn{3}{|c|}{$\boldsymbol{U}_{12}$} \\
\hline & $\delta$ & $\epsilon$ & $\alpha$ & $\delta$ & $-\epsilon$ & $\alpha$ & $-\delta$ & $\epsilon$ & $\alpha$ & $-\delta$ & $-\epsilon$ \\
\hline & o & $\epsilon$ & $\delta$ & $\alpha$ & $-\epsilon$ & $-\delta$ & $\alpha$ & $-\epsilon$ & $-\delta$ & $\alpha$ & $\epsilon$ \\
\hline & $\epsilon$ & $\beta$ & $-\epsilon$ & $-\epsilon$ & $\beta$ & $\epsilon$ & $-\epsilon$ & $\beta$ & $-\epsilon$ & $\epsilon$ & $\beta$ \\
\hline
\end{tabular}

Table 1: Transformation strains in the cubic to monoclinic-I transformation.

For $i \in\{3,4,5,9\}$, the condition (5.4) is found to be verified. Restriction to two wells is thus ensured for any $\overline{\boldsymbol{F}}$ in $Q\left(\mathrm{SO}(3) \boldsymbol{U}_{1} \cup \mathrm{SO}(3) \boldsymbol{U}_{i}\right)$.

For $i \in\{2,8,11\}$, neither (5.1) nor (5.4) is satisfied, so we turn our attention to (5.8) and (5.9) instead. To that purpose, the solutions of the twinning equation $\boldsymbol{R} \boldsymbol{U}_{i}-\boldsymbol{U}_{1}=\boldsymbol{a} \otimes \boldsymbol{n}$ are needed. That calculation is relatively simple if there exists a $180^{\circ}$ rotation $\boldsymbol{S}$ such that $\boldsymbol{U}_{i}=\boldsymbol{S}^{T} \boldsymbol{U}_{1} \boldsymbol{S}$ (Ericksen, 1981). In that case, the twinning equation $\boldsymbol{R} \boldsymbol{U}_{i}-\boldsymbol{U}_{1}=\boldsymbol{a} \otimes \boldsymbol{n}$ has two solutions, denoted by $\left(\boldsymbol{R}_{1}, \boldsymbol{a}_{1}, \boldsymbol{n}_{1}\right)$ and $\left(\boldsymbol{R}_{2}, \boldsymbol{a}_{2}, \boldsymbol{n}_{2}\right)$ and given by

$$
\begin{array}{lll}
\boldsymbol{a}_{1}=2\left(\frac{\boldsymbol{U}_{1}^{-1} \hat{\boldsymbol{e}}}{\left\|\boldsymbol{U}_{1}^{-1} \hat{\boldsymbol{e}}\right\|^{2}}-\boldsymbol{U}_{1} \hat{\boldsymbol{e}}\right) & , & \boldsymbol{n}_{1}=\hat{\boldsymbol{e}}, \\
\boldsymbol{a}_{2}=\rho \boldsymbol{U}_{1} \hat{\boldsymbol{e}} & , \quad \boldsymbol{n}_{2}=\frac{2}{\rho}\left(\hat{\boldsymbol{e}}-\frac{\boldsymbol{U}_{1}^{2} \hat{\boldsymbol{e}}}{\left\|\boldsymbol{U}_{1} \hat{\boldsymbol{e}}\right\|^{2}}\right),
\end{array}
$$

where $\hat{\boldsymbol{e}}$ is the axis of $\boldsymbol{S}$ and $\rho$ is taken such that $\left\|\boldsymbol{n}_{2}\right\|=1$. First consider the case $i=8$. It can be verified that $\boldsymbol{R} \boldsymbol{U}_{8}-\boldsymbol{U}_{1}=\boldsymbol{a} \otimes \boldsymbol{n}$ where $\boldsymbol{S}$ is the $180^{\circ}$ rotation of axis $\left(\boldsymbol{v}_{1}+\boldsymbol{v}_{2}\right) / \sqrt{2}$. For deformation gradients $\boldsymbol{U}_{1}+\lambda \boldsymbol{a}_{1} \otimes \boldsymbol{n}_{1}$ (resp. $\boldsymbol{U}_{1}+\lambda \boldsymbol{a}_{2} \otimes \boldsymbol{n}_{2}$ ), the condition (5.8) (resp. (5.9)) is found to be satisfied. Similar results are obtained for $i=11$.

The case $i=2$ is the least favorable in terms of restriction to two wells. Solutions of the twinning equation are given by the expressions (5.10) with $\hat{\boldsymbol{e}}=\boldsymbol{v}_{1}$. For deformation gradients $\boldsymbol{U}_{1}+\lambda \boldsymbol{a}_{1} \otimes \boldsymbol{n}_{1}$, we obtain that (5.8) is satisfied. However, for $\overline{\boldsymbol{F}}=\boldsymbol{U}_{1}+\lambda \boldsymbol{a}_{2} \otimes \boldsymbol{n}_{2}$, it appears that neither (5.8) nor (5.9) is satisfied. The reduction to two wells is thus established for only one of the two classes of first-rank laminates involving variants 1 and 2 .

One can repeat this analysis for all the pairs of variants. The results are collected in Table 2. The entry $r(i, j)$ in Table 2 is left blank if variants $i$ and 


\begin{tabular}{c|cccccccccccc} 
Variant & 1 & 2 & 3 & 4 & 5 & 6 & 7 & 8 & 9 & 10 & 11 & 12 \\
\hline 1 &. & 1 & 3 & 3 & 3 &. &. & 2 & 3 &. & 2 &. \\
2 & 1 &. & 3 & 3 &. & 2 & 3 &. &. & 2 &. & 3 \\
3 & 3 & 3 &. & 1 &. & 3 & 2 &. & 2 &. & 3 &. \\
4 & 3 & 3 & 1 &. & 2 &. &. & 3 &. & 3 &. & 2 \\
5 & 3 &. &. & 2 &. & 1 & 3 & 3 & 3 &. &. & 2 \\
6 &. & 2 & 3 &. & 1 &. & 3 & 3 &. & 2 & 3 &. \\
7 &. & 3 & 2 &. & 3 & 3 &. & 1 & 2 &. &. & 3 \\
8 & 2 &. &. & 3 & 3 & 3 & 1 &. &. & 3 & 2 &. \\
9 & 3 &. & 2 &. & 3 &. & 2 &. &. & 1 & 3 & 3 \\
10 &. & 2 &. & 3 &. & 2 &. & 3 & 1 &. & 3 & 3 \\
11 & 2 &. & 3 &. &. & 3 &. & 2 & 3 & 3 &. & 1 \\
12 &. & 3 &. & 2 & 2 &. & 3 &. & 3 & 3 & 1 &.
\end{tabular}

Table 2: Reduction to two wells in Ni-49.75Ti.

$j$ are not rank-1 connected. The entry $r(i, j)$ is set equal to 3 if reduction to two wells holds for any $\overline{\boldsymbol{F}}$ in $Q\left(\mathrm{SO}(3) \boldsymbol{U}_{i} \cup \mathrm{SO}(3) \boldsymbol{U}_{j}\right)$, i.e. for any deformation gradient $\overline{\boldsymbol{F}}$ that can be realized by a second-rank laminate involving variants $i$ and $j$. An entry $r(i, j)$ equal to 2 indicates that reduction to two wells is only ensured for deformation gradients $\overline{\boldsymbol{F}}$ that can be realized by a first-rank laminate. Lastly, the entry $r(i, j)$ is set equal to 1 if reduction to two wells only holds for one of the two types of first-rank laminates. The corresponding type of laminate can be characterized by the value of the normal $\boldsymbol{n}$ in the twinning equation. We have

$$
\begin{array}{ll}
\boldsymbol{n}=\boldsymbol{v}_{1} & \text { for }\{i, j\}=\{1,2\},\{3,4\} \\
\boldsymbol{n}=\boldsymbol{v}_{2} & \text { for }\{i, j\}=\{5,6\},\{7,8\} \\
\boldsymbol{n}=\boldsymbol{v}_{3} & \text { for }\{i, j\}=\{9,10\},\{11,12\}
\end{array}
$$

The numerical calculations have also been done for other compositions of NiTi alloys (Hane and Shield, 1999; Knowles and Smith, 1981) without observing any change to the results displayed in Table 2.

\subsection{Cubic to monoclinic-II transformation}

The twelve transformation strains for the cubic to monoclinic-II transformation are given in Table 3. Values of the lattice parameters are taken as $\alpha=0.9842, \beta=1.1042, \gamma=0.9178, \delta=0.016$. Those values correspond 


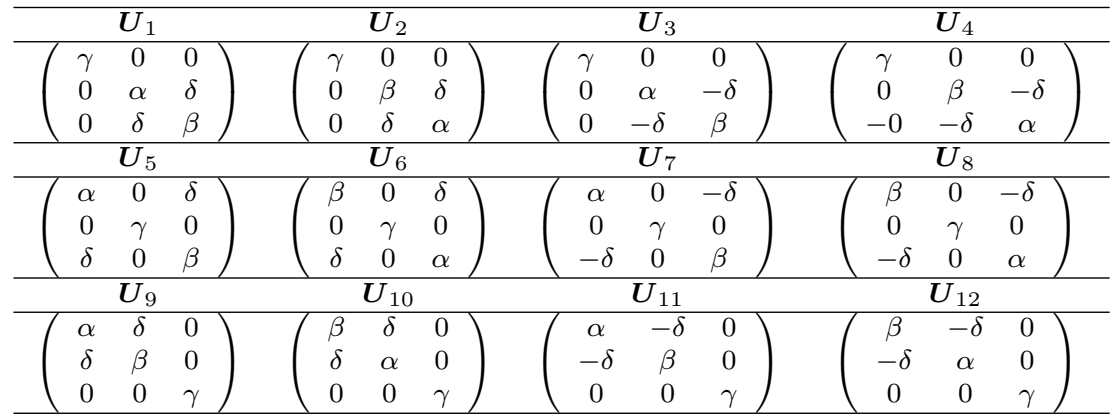

Table 3: Transformation strains in the cubic to monoclinic-II transformation.

to $\beta_{1}^{\prime} \mathrm{Cu}-14 \mathrm{Al}-4 \mathrm{Ni}$ (Otsuka et al., 1974). No pair of rank-1 connected variants satisfies (5.1) or (5.4). Therefore, contrary to the cubic to monoclinic I transformation, restriction to two wells can only be established for deformation gradients corresponding to first-rank laminates. Variant 1 is rank-1 connected with variant i for $i \in\{2,3,4,6,7,10,12\}$. For all those variants $\boldsymbol{U}_{i}$ except $\boldsymbol{U}_{4}$, there is a $180^{\circ}$ rotation $\boldsymbol{S}_{i}$ such that $\boldsymbol{S}_{i}^{T} . \boldsymbol{U}_{1} \cdot \boldsymbol{S}_{i}=\boldsymbol{U}_{i}$ (see e.g. Pitteri and Zanzotto (1998)). The solutions of the twinning equation are then given by expressions (5.10). Variant 1 is also rank- 1 connected with $\boldsymbol{U}_{4}$, but in that case there is no $180^{\circ}$ rotation $\boldsymbol{S}$ such that $\boldsymbol{S}^{T} \cdot \boldsymbol{U}_{1} \cdot \boldsymbol{S}=\boldsymbol{U}_{4}$. The solutions of the twinning equation can still be calculated, but the expressions are not as simple as (5.10) (see e.g. Ball and James (1987)).

For $i \in\{5,7\}$, we find - using (5.8) and (5.9) - that the restriction to two wells holds for any $\overline{\boldsymbol{F}}$ corresponding to a first-rank laminate.

For $i \in\{2,3\}$, use of (5.8) and (5.9) only ensures the restriction to two wells for one of the two solutions of the twinning equation $\boldsymbol{R} U_{i}-U_{1}=\boldsymbol{a} \otimes \boldsymbol{n}$, the corresponding value of $\boldsymbol{n}$ being given by

$$
\begin{array}{ll}
\boldsymbol{n}=\left(\boldsymbol{v}_{2}-\boldsymbol{v}_{3}\right) / \sqrt{2} & \text { for } i=2, \\
\boldsymbol{n}=\boldsymbol{v}_{2} & \text { for } i=3 .
\end{array}
$$

For $i \in\{4,10,12\}$, it is found that neither (5.8) nor (5.9) is satisfied. In that case, reduction to variants 1 and $i$ cannot be ensured, even though those two variants are rank-1 connected.

Table 4 summarizes the results obtained after going through all the pair of variants, using a similar nomenclature as in Table 2 . The entry $r(i, j)$ is set equal to 0 if variants $i$ and $j$ are rank-1 connected but no condition on $\overline{\boldsymbol{F}}$ has been found for restricting the microstructures to the two wells $\mathrm{SO}(3) \boldsymbol{U}_{i}$ 


\begin{tabular}{c|cccccccccccc} 
Variant & 1 & 2 & 3 & 4 & 5 & 6 & 7 & 8 & 9 & 10 & 11 & 12 \\
\hline 1 &. & 1 & 1 & 0 & 2 &. & 2 &. &. & 0 &. & 0 \\
2 & 1 &. & 0 & 1 &. & 0 &. & 0 & 2 &. & 2 &. \\
3 & 1 & 0 &. & 1 & 2 &. & 2 &. &. & 0 &. & 0 \\
4 & 0 & 1 & 1 &. &. & 1 &. & 1 & 2 &. & 2 &. \\
5 & 2 &. & 2 &. &. & 1 & 1 & 0 & 0 &. & 0 &. \\
6 &. & 0 &. & 0 & 1 &. & 0 & 1 &. & 2 &. & 2 \\
7 & 2 &. & 2 &. & 1 & 0 &. & 1 & 0 &. & 0 &. \\
8 &. & 0 &. & 0 & 0 & 1 & 1 &. &. & 2 &. & 2 \\
9 &. & 2 &. & 2 & 0 &. & 0 &. &. & 1 & 1 & 0 \\
10 & 0 &. & 0 &. &. & 2 &. & 2 & 1 &. & 0 & 1 \\
11 &. & 2 &. & 2 & 0 &. & 0 &. & 1 & 0 &. & 1 \\
12 & 0 &. & 0 &. &. & 2 &. & 2 & 0 & 1 & 1 &.
\end{tabular}

Table 4: Reduction to two wells in $\beta_{1}^{\prime} \mathrm{Cu}-14 \mathrm{Al}-4 \mathrm{Ni}$

and $\mathrm{SO}(3) \boldsymbol{U}_{j}$.

The results of Table 4 apply to $\beta_{1}^{\prime} \mathrm{Cu}-14 \mathrm{Al}-4 \mathrm{Ni}$. Using measurements of the lattice parameters available from the literature, we have also carried out the calculations for other materials undergoing a cubic to monoclinic-II transformation, such as Cu-15Zn-17Al (Chakravorty and Wayman, 1977), Cu-20Zn-12Ga (Saburi et al., 1976) or Cu-39.3Zn (Tadaki et al., 1975). The results are identical to those shown on Table 4.

It has to be emphasized that conditions (5.1)-(5.7) are sufficient conditions of $\overline{\boldsymbol{F}}$ for the reduction to two wells to hold. Therefore it is possible that the results of Tables 2 and 4 can be improved. For instance, for a pair of variants $\{i, j\}$ such that $r(i, j)=1$ in Table 2 or 4 , other arguments than (5.6)-(5.7) may show that restriction to two wells actually holds for any first-rank laminate of variants $i$ and $j$. It can be proved, however, that the values $r(i, j)$ in Table 4 are optimal for $(i, j) \in\{1,2,3,4\}^{2}$. Bhattacharya and Dolzmann (2001) indeed determined the quasiconvexification of the fourwell problem $\cup_{r=1}^{4} \mathrm{SO}(3) \boldsymbol{U}_{r}$ where $\left\{\boldsymbol{U}_{r}\right\}_{1 \leq r \leq 4}$ are given in Table 3 . Note that the four transformation gradients $\left\{\boldsymbol{U}_{r}\right\}_{1 \leq r \leq 4}$ have the same determinant and verify $U_{r} . \boldsymbol{v}_{1}=\gamma \boldsymbol{v}_{1}$. Taking this very special feature into account, Bhattacharya and Dolzmann determined the quasiconvex hull $Q\left\{\cup_{r=1}^{4} \mathrm{SO}(3) \boldsymbol{U}_{r}\right\}$ in closed form. They also obtained necessary and sufficient conditions for restriction to two wells to hold. Their results correspond to the predictions 
of Table 4: in the four-well problem $\cup_{r=1}^{4} \mathrm{SO}(3) \boldsymbol{U}_{r}$, restriction to two wells is only possible for the pair of variants $\{1,2\}$ and $\{1,3\}$, when in addition $\overline{\boldsymbol{F}}$ corresponds to one of the two types of first-rank laminate (given by (5.11)). It follows that the values of Table 4 for the twelve-well problem are optimal for $(i, j) \in\{1,2,3,4\}^{2}$. A similar conclusion holds for $(i, j) \in\{5,6,7,8\}^{2}$, and for $(i, j) \in\{9,10,11,12\}^{2}$.

Comparing Tables 2 and 4, we can see that stronger results are obtained for monoclinic-I martensite than for monoclinic-II martensite: for some pairs of variants in monoclinic-I martensite, reduction to two wells is ensured for any deformation gradient solution of the corresponding two-well problem. This is the strongest result that can be expected in terms of reduction to two wells, and such a result is not established for monoclinic-II martensite. On the other extreme, for monoclinic-II martensite, there are some pairs of rank1 connected variants for which restriction to two wells cannot be guaranteed (as indicated by the zero values in Table 4). That scenario does not occur for monoclinic-I martensite.

\section{Concluding remarks}

Considering the geometrically nonlinear setting, closed-form bounds have been obtained for a three-well problem that includes the cubic to tetragonal transformation as a special case. Although the exact expression of $Q \mathcal{K}$ remains out of reach, comparison with lower bounds obtained from lamination techniques shows that the upper bound $P \mathcal{K}$ is likely to be tight. It is not ensured that analog expressions can be derived for the cubic to orthorombic (or cubic to monoclinic) transformations, as calculation of the bound $P \mathcal{K}$ in (2.24) gets increasingly difficult as the number of variants grows. One can fall back on the bound $P_{0} \mathcal{K}$ in $(2.25)$ but it has been seen that some information is lost in that process.

It would be interesting to use such results to study recoverable strains of polycrystals. In that regard, a general method has been proposed by Peigney (2008) for bounding the recoverable strains of martensitic polycrystals, assuming that an upper bound on the set of recoverable strains in the constitutive reference single crystal is available. Combining that method with the results presented in this paper is the object of ongoing work.

Studying restrictions to four wells in cubic to monoclinic transformations has revealed some structural differences in the set of energy-minimizing strains corresponding to monoclinic-I and monoclinic-II martensite. Such 
differences will be further explored in Part 2 (Peigney, 2013), considering the geometrically linear setting.

\section{Appendix A. Proof of Theorem 4}

Consider a two-well problem $\mathcal{K}=\mathrm{SO}(3) \boldsymbol{U}_{1} \cup \mathrm{SO}(3) \boldsymbol{U}_{2}$ where $\left(\boldsymbol{U}_{1}, \boldsymbol{U}_{2}\right)$ are two symmetric positive tensors satisfying $\operatorname{det} \boldsymbol{U}_{1}=\operatorname{det} \boldsymbol{U}_{2}$ and $\boldsymbol{R} \boldsymbol{U}_{2}-\boldsymbol{U}_{1}=$ $\boldsymbol{a} \otimes \boldsymbol{n}$ for some rotation $\boldsymbol{R}$. In order to prove Theorem 4, we first need to express $Q \mathcal{K}$ in term of the canonical solution $Q \mathcal{K}^{0}$ in (3.6)-(3.7). This is accomplished by a change of variables introduced by Ball and James (1992). Define

$$
\begin{gathered}
\boldsymbol{e}_{1}=\frac{\boldsymbol{U}_{1}^{-1} \cdot \boldsymbol{n}}{\left\|\boldsymbol{U}_{1}^{-1} \cdot \boldsymbol{n}\right\|}, \boldsymbol{e}_{2}=\frac{\boldsymbol{a}}{\|\boldsymbol{a}\|}, \boldsymbol{e}_{3}=\boldsymbol{e}_{1} \wedge \boldsymbol{e}_{2}, \\
\delta=\frac{1}{2}\left\|\boldsymbol{U}_{1}^{-1} \cdot \boldsymbol{n}\right\| \cdot\|\boldsymbol{a}\|, \boldsymbol{L}=\boldsymbol{U}_{1}^{-1}\left(\boldsymbol{I}-\delta \boldsymbol{e}_{2} \otimes \boldsymbol{e}_{1}\right) .
\end{gathered}
$$

Since $\operatorname{det} \boldsymbol{U}_{1}=\operatorname{det} \boldsymbol{U}_{2}$, a simple calculation shows that $\boldsymbol{e}_{1} \cdot \boldsymbol{e}_{2}=0$, so that the basis $\left(\boldsymbol{e}_{1}, \boldsymbol{e}_{2}, \boldsymbol{e}_{3}\right)$ is orthonormal. It can be verified that

$$
\boldsymbol{U}_{1} \boldsymbol{L}=\boldsymbol{I}-\delta \boldsymbol{e}_{2} \otimes \boldsymbol{e}_{1}, \boldsymbol{U}_{2} \boldsymbol{L}=\boldsymbol{I}+\delta \boldsymbol{e}_{2} \otimes \boldsymbol{e}_{1}
$$

This construction can be applied to the tensors $\boldsymbol{U}_{1}^{0}$ and $\boldsymbol{U}_{2}^{0}$ in (3.6). Using expressions (5.10) and (A.1), we obtain that

$$
\boldsymbol{U}_{1}^{0} \boldsymbol{L}^{0}=\boldsymbol{I}-\delta^{0} \boldsymbol{e}_{2}^{0} \otimes \boldsymbol{e}_{1}^{0}, \boldsymbol{U}_{2}^{0} \boldsymbol{L}^{0}=\boldsymbol{I}+\delta^{0} \boldsymbol{e}_{2}^{0} \otimes \boldsymbol{e}_{1}^{0},
$$

with

$$
\begin{gathered}
\boldsymbol{e}_{1}^{0}=\frac{1}{\sqrt{\eta_{1}^{2}+\eta_{2}^{2}}}\left(\eta_{1} \boldsymbol{v}_{1}+\eta_{2} \boldsymbol{v}_{2}\right), \boldsymbol{e}_{2}^{0}=\frac{\operatorname{sgn}\left(\eta_{1}-\eta_{2}\right)}{\sqrt{\eta_{1}^{2}+\eta_{2}^{2}}}\left(\eta_{2} \boldsymbol{v}_{1}-\eta_{1} \boldsymbol{v}_{2}\right) \\
\delta^{0}=\frac{\left|\eta_{1}^{2}-\eta_{2}^{2}\right|}{2 \eta_{1} \eta_{2}}, \boldsymbol{L}^{0}=\left(\boldsymbol{U}_{1}^{0}\right)^{-1}\left(\boldsymbol{I}-\delta^{0} \boldsymbol{e}_{2}^{0} \otimes \boldsymbol{e}_{1}^{0}\right)
\end{gathered}
$$

Setting $\boldsymbol{v}_{3}=\boldsymbol{e}_{3}$, the expressions (A.2) show that it is always possible to choose $\left(\boldsymbol{v}_{1}, \boldsymbol{v}_{2}, \eta_{1}, \eta_{2}\right)$ such that $\boldsymbol{e}_{1}^{0}=\boldsymbol{e}_{1}, \boldsymbol{e}_{2}^{0}=\boldsymbol{e}_{2}$, and $\delta^{0}=\delta$. With such a choice, we find

$$
\boldsymbol{U}_{r} \cdot\left(\boldsymbol{U}_{1}^{-1} \boldsymbol{U}_{1}^{0}\right)=\boldsymbol{U}_{r}^{0}
$$

for $r=1,2$. Following Ball and James (1992), we have

$$
Q \mathcal{K} . \boldsymbol{U}_{1}^{-1} \boldsymbol{U}_{1}^{0}=Q \mathcal{K}^{0} .
$$


We are now in a position to prove Theorem 4. Consider indeed a given $\overline{\boldsymbol{F}}$ in $Q\left(\mathrm{SO}(3) \boldsymbol{U}_{1} \cup \mathrm{SO}(3) \boldsymbol{U}_{2}\right)$. By (A.4), there exists $\overline{\boldsymbol{F}}^{0} \in Q \mathcal{K}^{0}$ such that $\overline{\boldsymbol{F}} \boldsymbol{U}_{1}^{-1} \boldsymbol{U}_{1}^{0}=\overline{\boldsymbol{F}}^{0}$. Introduce the vector $\boldsymbol{k}$ defined by $\boldsymbol{k}=\boldsymbol{U}_{1}^{-1} \boldsymbol{U}_{1}^{0} \boldsymbol{e}_{3}=$ $\eta_{1} \boldsymbol{U}_{1}^{-1} \boldsymbol{e}_{3}$. Using (3.6) and (A.3), and recalling that $\boldsymbol{v}_{3}=\boldsymbol{e}_{3}$, it can be easily calculated that

$$
\boldsymbol{U}_{1} \cdot \boldsymbol{k}=\boldsymbol{U}_{2} \cdot \boldsymbol{k}=\eta_{1} \boldsymbol{e}_{3},\|\overline{\boldsymbol{F}} \cdot \boldsymbol{k}\|=\eta_{1} .
$$

Let us now verify that $\boldsymbol{k} \in \operatorname{Ker}\left(\boldsymbol{U}_{1}^{2}-\boldsymbol{U}_{2}^{2}\right)$. Using (3.2), we find

$$
\boldsymbol{U}_{2}^{2}-\boldsymbol{U}_{1}^{2}=\|\boldsymbol{a}\|^{2} \boldsymbol{n} \otimes \boldsymbol{n}+\boldsymbol{U}_{1} . \boldsymbol{a} \otimes \boldsymbol{n}+\boldsymbol{n} \otimes \boldsymbol{U}_{1} . \boldsymbol{a},
$$

hence $\left(\boldsymbol{U}_{2}^{2}-\boldsymbol{U}_{1}^{2}\right) \cdot \boldsymbol{k}=(\boldsymbol{n} \cdot \boldsymbol{k})\left(\|\boldsymbol{a}\|^{2} \boldsymbol{n}+\boldsymbol{U}_{1} \boldsymbol{a}\right)+\left(\boldsymbol{k} \cdot \boldsymbol{U}_{1} \cdot \boldsymbol{a}\right) \boldsymbol{n}$. The definitions (A.1) give

$$
\boldsymbol{n} . \boldsymbol{k}=\eta_{1}\left\|\boldsymbol{U}_{1}^{-1} \cdot \boldsymbol{n}\right\| \boldsymbol{e}_{1} \cdot \boldsymbol{e}_{3}=0
$$

and

$$
\boldsymbol{k} . \boldsymbol{U}_{1} \cdot \boldsymbol{a}=\eta_{1}\|\boldsymbol{a}\| \boldsymbol{e}_{2} \cdot \boldsymbol{e}_{3}=0 .
$$

Therefore $\boldsymbol{k} \in \operatorname{Ker}\left(\boldsymbol{U}_{1}^{2}-\boldsymbol{U}_{2}^{2}\right)$. It remains to observe that under assumptions (3.1), the dimension of $\operatorname{Ker}\left(\boldsymbol{U}_{1}^{2}-\boldsymbol{U}_{2}^{2}\right)$ is necessarily equal to 1 . Indeed, if $\operatorname{Ker}\left(\boldsymbol{U}_{1}^{2}-\boldsymbol{U}_{2}^{2}\right)$ was of dimension 2, there would exist a unit vector $\boldsymbol{b}$ and a scalar $\gamma \neq 0$ such that $\boldsymbol{U}_{1}^{2}-\boldsymbol{U}_{2}^{2}=\gamma \boldsymbol{b} \otimes \boldsymbol{b}$. This relation can be rewritten as $\boldsymbol{U}_{1}^{2}=\boldsymbol{U}_{2}\left(\boldsymbol{I}+\gamma \boldsymbol{U}_{2}^{-1} \boldsymbol{b} \otimes \boldsymbol{U}_{2}^{-1} \boldsymbol{b}\right) \boldsymbol{U}_{2}$. Since $\operatorname{det} \boldsymbol{U}_{1}=\operatorname{det} \boldsymbol{U}_{2}$, we find that $\operatorname{det}\left(\boldsymbol{I}+\gamma \boldsymbol{U}_{2}^{-1} \boldsymbol{b} \otimes \boldsymbol{U}_{2}^{-1} \boldsymbol{b}\right)=1$. This last equality can be satisfied only if $\gamma \boldsymbol{U}_{2}^{-1} \boldsymbol{b}=0$, thus contradicting the fact that $\gamma \neq 0$ and $\|\boldsymbol{b}\|=1$. Therefore, the dimension of $\operatorname{Ker}\left(\boldsymbol{U}_{1}^{2}-\boldsymbol{U}_{2}^{2}\right)$ is equal to 1, so that any $\boldsymbol{v}$ in $\operatorname{Ker}\left(\boldsymbol{U}_{1}^{2}-\boldsymbol{U}_{2}^{2}\right)$ is colinear to $\boldsymbol{k}$. The equality $\|\overline{\boldsymbol{F}} \cdot \boldsymbol{v}\|=\left\|\boldsymbol{U}_{1} \cdot \boldsymbol{v}\right\|$ thus follows directly from (A.5).

Setting $\boldsymbol{k}^{\prime}=\left(\boldsymbol{U}_{1}^{*}\right)^{-1}\left(\boldsymbol{U}_{1}^{0}\right)^{*} \boldsymbol{e}_{3}$, a similar reasoning shows that

$$
\boldsymbol{U}_{1}^{*} \cdot \boldsymbol{k}^{\prime}=\boldsymbol{U}_{2}^{*} \cdot \boldsymbol{k}^{\prime}=\eta_{1} \eta_{2} \boldsymbol{e}_{3},\left\|\overline{\boldsymbol{F}}^{*} \cdot \boldsymbol{k}^{\prime}\right\|=\eta_{1} \eta_{2},\left(\boldsymbol{U}_{2}^{*, 2}-\boldsymbol{U}_{1}^{*, 2}\right) \cdot \boldsymbol{k}=0 .
$$

Using the twinning equation (3.2) as well the relation $\operatorname{det} \boldsymbol{U}_{1}=\operatorname{det} \boldsymbol{U}_{2}$, a simple calculation shows that

$$
\boldsymbol{R} \boldsymbol{U}_{2}^{*}-\boldsymbol{U}_{1}^{*}=-\left(\operatorname{det} \boldsymbol{U}_{1}\right)\left(\boldsymbol{R} \boldsymbol{U}_{2}\right)^{-T} \boldsymbol{n} \otimes \boldsymbol{U}_{1}^{-1} \boldsymbol{a} .
$$

The sets $\mathrm{SO}(3) \boldsymbol{U}_{1}^{*}$ and $\mathrm{SO}(3) \boldsymbol{U}_{2}^{*}$ are thus rank-1 connected. One can show as above that $\operatorname{Ker}\left(\boldsymbol{U}_{1}^{*, 2}-\boldsymbol{U}_{*, 2}^{2}\right)$ is of dimension 1 and therefore conclude in a similar way. 


\section{References}

Ball, J., James, R., 1987. Fine phase mixtures as minimizers of energy. Arch. Rational Mech. Anal. 100, 13-52.

Ball, J., James, R., 1992. Proposed experimental tests of a theory of fine microstructure and the two-well problem. Phil. Trans. Roy. Soc. London A. $338,338-450$.

Bhattacharya, K., Dolzmann, G., 2001. Relaxation on some multi-well problems. Proc. Royal Soc. Edin A 131, 279-320.

Bhattacharya, K., Kohn, R., 1997. Energy minimization and the recoverable strains in polycrystalline shape memory alloys. Arch. Rational Mech. Anal. 139, 99-180.

Bhattacharya, K., Li, B., Luskin, M., 1999. The simply laminated microstructure in martensitic crystals that undergo a cubic to orthorombic phase transformation. Arch. Rational Mech. Anal. 149, 123-154.

Chakravorty, S., Wayman, C., 1977. Electron microscopy of internally faulted CuZnAl martensite. Acta Metall. 25, 989-1000.

Dacorogna, B., 2008. Direct methods in the calculus of variations, second edition. Springer.

Dolzmann, G., 1999. Numerical computation of rank-one convex envelopes. SIAM J. Numer. Anal 36, 1621-1635.

Ericksen, J., 1981. Continuous martensitic transitions in thermoelastic solids. J. Thermal Stresses 4, 107-119.

Hane, K., Shield, T., 1999. Microstructure in the cubic to monoclinic transition in titanium-nickel shape memory alloys. Acta Mater. 47, 2603-2617.

Kinderlehrer, D., Pedregal, P., 1991. Characterizations of young measures generated by gradients. Arch. Rational Mech. Anal. 115, 329-365.

Knowles, K., Smith, D., 1981. Crystallography of the martensitic transformation in equiatomic nickel-titanium. Acta Mater. 29, 101-110. 
Kohn, R., 1991. Relaxation of a double-well energy. Continuum Mech. Thermodyn. 3, 193-236.

Müller, S., 1999. Variational models for microstructure and phase transitions. In: Calculus of variations and geometric evolution problems. Springer.

Otsuka, K., Nakamura, T., Shimizu, K., 1974. Electron microscopy study of stress-induced acicular $\beta_{1}^{\prime}$ martensite in $\mathrm{Cu}-\mathrm{Al}-\mathrm{Ni}$ alloy. Trans. Jap. Inst. Metals 15, 225-237.

Peigney, M., 2008. Recoverable strains in composite shape-memory alloys. J. Mech. Phys. Solids 56, 360-375.

Peigney, M., 2013. On the energy-minimizing strains in martensitic microstructures - Part 2: Geometrically linear theory. J. Mech. Phys. Solids 61, 1511-1530.

Pitteri, M., Zanzotto, G., 1998. Generic and non-generic cubic-to-monoclinic transitions and their twins. Acta Mater. 46, 200-210.

Saburi, T., Nenno, S., Kato, S., Takata, K., 1976. Configurations of martensite variants in $\mathrm{Cu}-\mathrm{Zn}-\mathrm{Ga}$. J. Less Common Metals 50, 223-236.

Smyshlyaev, V., Willis, J., 1998. On the relaxation of a three-well energy. Proc. R. Soc. Lond. A 455, 779-814.

Stupkiewicz, S., Petryk, H., 2002. Modelling of laminated microstructures in stress-induced martensitic transformations. J. Mech. Phys. Solids 50, $2303-2331$.

Tadaki, T., Tokoro, M., Shimizu, K., 1975. Thermoelastic nature and crystal structure of the $\mathrm{Cu}-\mathrm{Zn}$ martensite related to the shape memory alloy. Trans. Jap. Inst. Metals 56, 285-296. 